\title{
The Fossil Wind Structures of Eta Carinae: Changes across one 5.54-year Cycle
}

\author{
Theodore R. Gull, ${ }^{1}{ }^{\star}$ Thomas I. Madura, ${ }^{1,2}$ Mairan Teodoro, ${ }^{1,2}$ \\ Nicola Clementel, ${ }^{3}$ Michael Corcoran, ${ }^{1,2}$ Augusto Damineli, ${ }^{4}$ Jose H. Groh, ${ }^{5}$ \\ Kenji Hamaguchi, ${ }^{1,6}$ D. John Hillier, ${ }^{7}$ Anthony F. J. Moffat, ${ }^{8}$ \\ Noel D. Richardson, ${ }^{9}$ Gerd Weigelt, ${ }^{10}$ \\ Don Lindler, ${ }^{1,11}$ Keith Feggans ${ }^{11,12}$ \\ ${ }^{1}$ Code 660, Astrophysics Science Division, Goddard Space Flight Center, Greenbelt, MD 20771 USA \\ ${ }^{2}$ Universities Space Research Association, 7178 Columbia Gateway Drive, Columbia, MD 20146 USA \\ ${ }^{3}$ South African Astronomical Observatory, P. O. Box 9, Observatory 7935, South Africa \\ ${ }^{4}$ Instituto de Astronomia, Geofisica e Ciencias Atmosfericas, Universidade de Sao Paulo, Rua do Matao 1226, \\ Cidade Universitaria, Sao Paulo 05508-900, Brazil \\ ${ }^{5}$ School of Physics, Trinity College Dublin, The University of Dublin, Dublin 2, Ireland \\ ${ }^{6}$ Department of Physics, University of Maryland, Baltimore County, 1000 Hilltop Circle, Baltimore, MD 21250, USA \\ ${ }^{7}$ Department of Physics and Astronomy and Pittsburgh Particle physics, Astrophysics, and Cosmology Center (PITT PACC), \\ University of Pittsburgh, 3941 OHara Street, Pittsburgh, PA 15260, USA \\ ${ }^{8}$ Departement de physique and Centre de Recherche en Astrophysique du Quebec, Universite de Montreal, CP 6128 Succ. A., \\ Centre-Ville, Montreal, Quebec H3C 3J7, Canada \\ ${ }^{9}$ Ritter Observatory, Department of Physics and Astronomy, The University of Toledo, Toledo, OH 43606-3390, USA \\ ${ }^{10}$ Max-Planck-Institut fur Radioastronomie, Auf dem Hugel 69, 53121, Bonn, Germany \\ ${ }^{11}$ Sigma Space Corporation, 4600 Forbes Blvd., Lanham, MD 20706 USA \\ ${ }^{12}$ Code 670, Heliophysics Science Division, Goddard Space Flight Center, Greenbelt, MD 20771 USA
}

Accepted XXX. Received YYY; in original form ZZZ

\begin{abstract}
Eta Carinae, the closest, active, massive binary containing a highly unstable LBV, exhibits expanding, compressed wind shells, seen in emission, that are spatially and spectrally resolved by HST/STIS. Starting in June 2009, these structures were mapped across its 5.54-year, highly elliptical, binary orbit to follow temporal changes in the light of [Fe III] $4659 \AA$ and [Fe II] $4815 \AA$. The emissions trace portions of fossil windshells, that were formed by wind-wind interactions across each cycle. Over the highionization state, dense arcs, photo-ionized by far ultraviolet radiation from the hot secondary, are seen in [Fe III]. Other arcs, ionized by mid-ultraviolet radiation from the primary star, are seen in $[\mathrm{Fe} \mathrm{II}]$. The $[\mathrm{Fe} \mathrm{III]} \mathrm{structures} \mathrm{tend} \mathrm{to} \mathrm{be} \mathrm{interior} \mathrm{to}$ [Fe II] structures that trace extensive, less disturbed primary wind. During the brief periastron passage when the secondary plunges deep into the primary's extremely dense wind, on the far side of primary star, high-ionization [Fe III] structures fade and reappear in [Fe II]. Multiple fossil wind-structures were traced across the 5.7year monitoring interval. The strong similarity of the expanding [Fe II] shells suggests that the wind and photo-ionization properties of the massive binary have not changed substantially from one orbit to the next over the past several orbital cycles. These observations trace structures that can be used to test three-dimensional hydrodynamical and radiative-transfer models of massive, interacting winds. They also provide a baseline for following future changes in $\eta$ Car, especially of its winds and photoionization properties.
\end{abstract}

Key words: stars: atmospheres — stars: mass-loss — stars: variables: general — supergiants — stars: individual (Eta Carinae)

* E-mail: Theodore.R.Gull@nasa.gov

(C) 2016 The Authors 


\section{INTRODUCTION}

The massive binary system, Eta Carinae $(\eta \text { Car })^{1}$, has fascinated astronomers since it brightened in the 1840s to rival Sirius, faded below threshold of the unaided eye, brightened again in the 1890s, and again faded (Davidson \& Humphreys 1997). In recent decades, $\eta$ Car has gradually brightened to naked-eye visibility ${ }^{2}$ (Fernández-Lajús et al. 2009). $\eta$ Car is sufficiently nearby ( $D=2.3 \pm 0.1 \mathrm{kpc}$, Walborn 2012) that associated structures can be studied with moderate spatial resolution throughout the electromagnetic spectrum. Damineli (1996) discovered a period of 5.5 years based upon the periodic modulation of the He I $10830 \AA^{3}$ plus other He I, [Ne III], [Ar III] and [Fe III] lines. He suggested a massive primary, $\eta$ Car A, with a hotter, less massive, secondary star, $\eta$ Car B, in a highly eccentric orbit, could account for the observed high- and low-ionization states. While Damineli et al. (1997) estimated the binary orbit eccentricity to be 0.63, Davidson (1997) suggested that eccentricity had to be at least 0.8 while Corcoran et al. (2001) derived an even higher $\mathrm{e}=0.9$.

Pittard \& Corcoran (2002) modeled the X-ray spectrum obtained with the Chandra X-ray grating and determined that the wind of $\eta$ Car B required terminal velocity, $v_{\infty, B}=$ $3000 \mathrm{~km} \mathrm{~s}^{-1}$, with a mass loss rate, $\dot{M}_{B}=10^{-5} M_{\odot} \mathrm{yr}^{-1}$ while the wind of the much more massive star, $\eta$ Car A, was in the range of $v_{\infty, A}=500$ to $700 \mathrm{~km} \mathrm{~s}^{-1}$ and $\dot{M}_{A}=$ $2.4 \times 10^{-4} M_{\odot} \mathrm{yr}^{-1}$. Okazaki et al. (2008) were able to reproduce the Rossi X-ray Telescope Explorer (RXTE) X-ray light curve (Corcoran et al. 2001; Corcoran 2005) using an orbital eccentricity of 0.9 . Parkin et al. (2009) varied the eccentricity and wind parameters and concluded that an eccentricity close to 0.9 , but likely less than 0.95 , fitted the RXTE X-ray light curve published by Corcoran (2005). While the best estimates of the secondary wind have not changed, improved models for $\eta$ Car A's spectrum suggest $v_{\infty, A}=420 \mathrm{~km} \mathrm{~s}^{-1}$ and $\dot{M}_{A}=8.5 \times 10^{-4} M_{\odot} \mathrm{yr}^{-1}$ (Groh et al. 2012).

Previous speckle interferometry of $\eta$ Car by Weigelt \& Ebersberger (1986) and speckle masking by Hofmann \& Weigelt (1988) resolved four point-like sources within $0^{\prime \prime} 3$ of each other (called Weigelt A, B, C and D). Weigelt et al. (1995) used Hubble Space Telescope/Faint Object Camera $H S T / F O C$ observations to confirm the existence of these four objects in the UV as well as the visible regions. Falcke et al. (1996) obtained speckle polarimetric imagery at $\mathrm{H} \alpha$ of $\eta$ Car that were consistent with an elongated, disklike structure extending from northeast to southwest across $\eta$ Car. Davidson et al. (1995a), using the HST/ Faint Object Spectrograph (HST/FOS), determined that Weigelt A is a stellar source, but Weigelt B, C and D proved to be extraordinarily bright emission-line sources located within 0.3 (projected $690 \mathrm{AU}$ ) of $\eta \mathrm{Car}^{4}$.

1 Based on observations made with the NASA/ESA Hubble Space Telescope. Support for Program numbers 12013, 12750, 12508,13054 and 13395 was provided through grants from the Space Telescope Science Institute, which is operated by the Association of Universities for Research in Astronomy, Incorporated, under NASA contract NAS5-26555.

2 See photometry at http://etacar.fcaglp.unlp.edu.ar/

3 All wavelengths in this article are referenced to vacuum.

4 Teodoro et al. (in prep) demonstrate that the Weigelt ob-
Discovery of the 5.5-year period by Damineli (1996) coincided with installation of the Space Telescope Imaging Spectrograph (STIS) in HST, which enabled near diffractionlimited spatial resolution $(0.1$ at $5000 \AA)$ spectroscopic studies at a selection of optimal spectral resolving powers ranging from a few hundred to a few hundred thousand (Kimble et al. 1998; Woodgate et al. 1998). Zethson (2001) and Zethson et al. (2012) used HST/STIS spatially resolved, time variable spectra of Weigelt $B$ and $D$ to identify a few thousand nebular lines primarily of ionized iron-peak elements. During the 5.2-year, high-ionization state, emission lines from many species, most notably iron-peak elements were identified originating from singly to triply ionized states. Strong lines of [Ne III] demonstrated that far ultraviolet (FUV) emission, with energies exceeding $40 \mathrm{eV}$, excited the Weigelt clumps. However, during the monthslong low-ionization state, the FUV radiation drops and the spectrum is dominated by singly ionized species.

Observations in the ultraviolet were first accomplished with the International Ultraviolet Explorer (IUE) (Heap et al. 1978) and extended by HST/STIS (Nielsen et al. 2005; Gull et al. 2006) and the Far Ultraviolet Spectroscopic Explorer (FUSE) (Iping et al. 2005). Both the HST and FUSE observations confirmed that the low-ionization state was indeed brought about by a drop in the ultraviolet flux most noticeably below $1500 \AA$. Indeed, the nearly one thousand ultraviolet narrow absorption lines of molecular hydrogen seen at $-512 \mathrm{~km} \mathrm{~s}^{-1}$ across the high-ionization state, due to the expanding Homunculus, disappeared during the 2003.5 low-ionization state and reappeared as the high-ionization state again resumed (Nielsen et al. 2005). Hillier et al. (2001, 2006) modeled the HST/STIS and FUSE observations showing that while the spectrum of the primary star dominates from the FUV to longer wavelengths, the intrinsic obscuration in the $\eta$ Car system and the greatly extended primary wind prevented direct detection of the secondary companion. Hence while the periodic modulation of the X-rays, the visible-wavelength broad emission lines and the FUV demonstrate the presence of a hot secondary companion, direct detection is yet to be accomplished.

A very peculiar property of $\eta$ Car's spectrum is the presence of both narrow ( $\left.\approx 40 \mathrm{~km} \mathrm{~s}^{-1} \mathrm{FWHM}\right)$ and broad components $\left(\approx 900 \mathrm{~km} \mathrm{~s}^{-1}\right)$ for many permitted and forbidden emission lines as measured by seeing-limited $\left(1-2^{\prime \prime}\right)$ ground-based spectroscopy. Spectra recorded by $H S T / S T I S$ with the $52^{\prime \prime} \times \quad 0^{\prime \prime} 1$ aperture showed a broad line profile at the stellar position and a narrow line profile originating from Weigelt objects C and D (Gull et al. 2009). However weak narrow, velocity-shifted components could be seen extending beyond $\eta$ Car at various position angles (PAs). The strengths of these components changed between $H S T / S T I S$ observations recorded at different orbital phases of $\eta$ Car across the interval from 1998.0 to 2004.3.

Also peculiar is the anomalous reddening towards $\eta$ Car (Hillier et al. 2001). In addition to normal interstellar reddening in the direction of the Carinae complex, a relatively grey extinction is thought to originate within the Homuncu-

jects are UV-illuminated surfaces of large, massive, slowly-moving clumps of infrared-bright material noticed by Chesneau et al. (2005). 
lus and more likely close to the star. Curiously this extinction does not appear in the spectra of the Weigelt clumps (Gull et al. 2009; Mehner et al. 2010), indicating that the grey extinction possibly is circumstellar.

Extensive reviews of the many HST/STIS longslit spectra of $\eta$ Car, recorded from 1998 to 2004, led to the realization that extended wind structures could be traced along multiple PAs, and that the contributions of low- and highionization forbidden lines (e.g. [Ne III], [Fe III], [Ar III], [Fe II] and [Ni II]) changed with binary phase (Gull et al. 2009). However, a controller board in the STIS failed in 2004, so no further spectroscopic studies could be done with the HST until repairs were accomplished during the Servicing Mission 4 in May 2009. High spatial resolution, combined with moderate spectral resolution, using ground-based facilities was, and continues to be, not possible as adaptive optics correction, coupled with moderate spectral resolution $(\mathrm{R} \approx 10,000)$, does not yet extend into the visible spectral region.

The HST/STIS longslit spectra and HST/Wide Field Planetary Camera 2 (WFPC2) direct imagery revealed that the Homunculus, ejecta from the 1840s Great Eruption of $\eta$ Car, is a bipolar structure tilted about 45 degrees into the sky plane (Davidson et al. 2001). However, it was not obvious that the binary orbital plane was, or was not, aligned with the Homunculus axis of symmetry. Three-dimensional (3-D) modeling of the X-ray light curve led to evidence that the axis of the orbital plane was tilted at about $45^{\circ}$ into the sky plane, but a degeneracy existed as to which direction the tilt axis was oriented within the sky plane (Okazaki et al. 2008). Improved 3-D modeling (Madura 2012) combined with the HST/STIS longslit observations showed that the normal to the binary orbital plane was closely aligned to the Homunculus major axis of symmetry, thereby suggesting the possibility that formation of the Homunculus was influenced by the binary system (Gull et al. 2009). The 3D Homunculus model of $\mathrm{H}_{2}$ near infrared emission strongly supports this idea (Steffen et al. 2014) .

Many changes with binary phase are apparent in the spectra. The most prominent is the disappearance of the high-ionization emission $(>13.6 \mathrm{eV})$ across the periastron and with the appearance of low-ionization emission $(<13.6$ $\mathrm{eV})$. These resolved emission structures are the result of not just the current wind-wind interactions, but also wind-wind interactions from the most recent cycles. Their visibility is the result of absence or presence of FUV radiation impinging upon structures with relatively high electron densities $(\approx$ $10^{7} \mathrm{~cm}^{-3}$ ). These structures are the compressed, expandingwind structures that persist for more than one 5.5-year cycle. Hence we call them 'fossil wind' structures. They provide a historical record of photoionization, expansion, and density over the several most recent cycles (Teodoro et al. 2013).

Many observations accomplished over the past few decades show that $\eta$ Car is a unique, massive binary system:

(i) The interacting winds are so massive that the forbidden lines from $\mathrm{Fe}^{++}$and $\mathrm{Fe}^{+}$trace portions of dense fossil shells (Gull et al. 2009).

(ii) The orbit is sufficiently eccentric that the hot secondary spends considerable time near its apastron position, effectively clearing a large cavity out of the primary wind. (iii) The high-ionization emission is mostly blue-shifted while the low-ionization emission is mostly red-shifted, which supports 3-D models (Gull et al. 2009; Madura 2012) with apastron occurring with $\eta$ Car B positioned on the near side of $\eta$ Car A. Periastron occurs with $\eta$ Car B on the far side of $\eta$ Car A (Gull et al. 2011).

These fossil shells provide powerful clues to the physics of the wind-wind interactions, the orientation of the binary orbit and provide a measure of changes in the properties of the winds over several cycles.

The HST/STIS spatially-resolved spectra centered on $\eta$ Car recorded prior to 2004 provided individual slices of the 3-D fossil wind structures at a number of orbital phases but were insufficient to describe the entire structure and the dynamical changes across the 5.54-year cycle (Gull et al. 2009). Mapping was needed since single spectra at arbitrary roll angles at different orbital phases could not distinguish spatial- from phase-variability. We determined that mapping the structures revealed by selected forbidden emission lines with the HST/STIS across the central $2^{\prime \prime} \times 2^{\prime \prime}$ at selected phases could provide much insight on the density and ionization conditions of these expanding shells partially photo-ionized by the FUV radiation from $\eta$ Car B.

The spatial mappings of the central region surrounding $\eta$ Car have already proven to be very useful for related studies:

(i) Observations recorded from 2009 through 2013 have been used to measure expansion of structures seen in [Fe II] and [Ni II] (Teodoro et al. 2013). These low-ionization structures are red-shifted, moving at $470 \mathrm{~km} \mathrm{~s}^{-1}$ and are associated with the primary wind. Gaps between the shells are due to the passage of the hot secondary across the periastron event that occurs every 5.54 years. While the primary wind velocity was measured to be $420 \mathrm{~km} \mathrm{~s}^{-1}$ (Groh et al. 2012), these shells expand at a slightly higher velocity possibly due to acceleration by the much faster secondary wind.

(ii) The $4706 \AA$ grating setting included the He II $4686 \AA$ line that dramatically strengthens as the stars approach periastron (Steiner \& Damineli 2004). Teodoro et al. (2016) resolved the apparent discrepancy between published groundbased and HST/STIS measures (Davidson et al. 2015). The He II emission comes from within $0 . \prime 13$ centered on $\eta$ Car, but seeing-limited ground-based spectroscopy includes $1-2^{\prime \prime}$ of nebular structure, leading to increased continuum and a smaller He II $4686 \AA$ equivalent width measured from the ground.

(iii) Studies of He I 4714, 5876 and $7065 \AA$ line profile variations with ground-based, high-dispersion $(\mathrm{R}=\lambda / \delta \lambda=$ $90,000)$ spectra were shown to be consistent with spectral profiles integrated from $H S T / S T I S$ mappings in the same time frame (Richardson et al. 2016). While most of the He I line profiles come from the central $0^{\prime \prime} 13$ core, the line profiles as observed from the ground are considerably modified by absorptions and scattered light from the fossil wind structures plus slowly moving debris (the Weigelt blobs) from the two historical ejections.

These observations show quite complex structures that change continuously across the 5.54-year binary cycle. Patterns emerge that provide clues to how the interacting winds are shaping their environment, including interactions with 
slowly moving clumps. Explanation demands 3-D modeling efforts to replicate the fossil wind structures. Models fitted to the observations will improve constraints on the stellar winds and the binary orientation parameters, possibly even new insights on the physics of the outbursts.

Preliminary hydrodynamic models with limited radiative transfer have already been accomplished by Madura et al. (2013) and Clementel et al. (2014, 2015a,b). Additional observations at higher angular resolution in the near infrared and radio will provide considerable new information. Specifically, observations with the Very Large Telescope Interferometer (VLTI) with angular resolutions of a few milliarcseconds are already revealing information on the current interacting wind properties (Weigelt et al. submitted).

We describe the observations in Section 2. Changes in structure across the series of mappings are described in Section 3. Discussion of these changes and implications on the properties of the two massive components are presented in Section 4 with conclusions in Section 5. Details of the data reduction are presented in Appendix A. Two very large mosaics of [Fe III] and [Fe II] showing the iso-velocity slices for mappings are presented in Appendix B. while a discussion of the changing fluxes is given in Appendix $\mathrm{C}$.

\section{OBSERVATIONS}

The first mapping observations for this study were done with the HST/STIS in June 2009 across the $2^{\prime \prime} \times 2^{\prime \prime}$ region centered upon $\eta$ Car with the $52^{\prime \prime} \times 00^{\prime \prime} 1$ aperture at 0.1 spacing (230 AU at $2.3 \mathrm{kpc}$ distance) for several grating settings including the G430M grating centered at $4706 \AA$ (Noll 2009). Each of the grating settings had been selected to map specific forbidden lines that would reveal a range of ionization states. We had reviewed the line list, provided by Zethson (2001), searching for strong forbidden lines that were minimally contaminated by other emission lines or absorptions from strong wind lines such as He I $4714 \AA$, which is on the red side of [Fe III] $4701 \AA$. Two forbidden lines of interest, [Fe III] $4659.35 \AA$ and [Fe II] $4815.88 \AA$, both of which are relatively isolated from wind and nebular lines, proved to be excellent candidates as they originate from the same atomic species and provide ionization information above $(16.2 \mathrm{eV})$ and below $(7.9 \mathrm{eV})$ the ionization potential of hydrogen $(13.6$ $\mathrm{eV})$.

Other grating settings in the initial visits provided map-

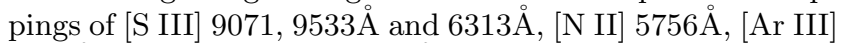

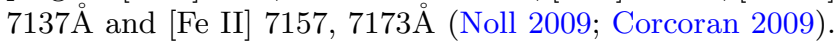
The [S III] mapping proved to be of less utility due to the STIS CCD performance and the broadened HST point spread function in the near red. Given the very limited $H S T$ observing time, we streamlined the full cycle program to obtain maps of [Fe III] $4659 \AA$ and [Fe II] $4815 \AA$ at selected phases, but we added maps of [N II] $5756 \AA$ and [Ar III] $7137 \AA$ at phases when considerable changes in ionization were predicted by the 3 -D models (Madura 2010). This article focuses on the changes of [Fe III] $4659 \AA$ and [Fe II] $4814 \AA$, herein labeled [Fe III] and [Fe II], across a full orbital cycle. Mappings of [N II], [Ar III] and He I will be presented in other papers.

The first STIS mapping occurred five months after the 2009.0 periastron event and confirmed that $\eta$ Car and the
Table 1. HST/STIS Data Used in This Analysis

\begin{tabular}{|c|c|c|c|c|}
\hline $\begin{array}{l}H S T \text { Obs. } \\
\text { Prog }\end{array}$ & Obs. Date & $\begin{array}{l}\text { Orbital }^{a} \\
\text { Phase }\end{array}$ & $\begin{array}{l}\text { Slit PA } \\
\text { Degrees }\end{array}$ & $\begin{array}{l}\text { Mapped } \\
\text { Region }^{b, c, d}\end{array}$ \\
\hline $11506^{e}$ & Jun 29, 2009 & 12.08408 & +79.5 & $3^{\prime \prime} 2 \times 2^{\prime \prime} 0$ \\
\hline $12013^{e}$ & Dec 6, 2009 & 12.16318 & -121.0 & $3^{\prime \prime} .2 \times 1^{\prime \prime} 4$ \\
\hline $12013^{e}$ & Oct 10,2010 & 12.31545 & -166.7 & $6 . .4 \times 1^{\prime \prime} .1$ \\
\hline 12508 & Nov 20, 2011 & 12.51617 & -138.7 & $6 .^{\prime \prime} 4 \times 22^{\prime \prime} 0$ \\
\hline 12750 & Oct 18,2012 & 12.68080 & -174.8 & $6 . .4 \times 22^{\prime \prime} 0$ \\
\hline 13054 & Sep 32013 & 12.83901 & +136.7 & $6 !^{\prime \prime} 4 \times 11^{\prime \prime} 8$ \\
\hline 13395 & Feb 17, 2014 & 12.92157 & +169.2 & $6 . .4 \times 1^{\prime \prime} .9$ \\
\hline 13395 & Jun 9,2014 & 12.97694 & +61.4 & $6 .^{\prime \prime} 4 \times 2 .^{\prime \prime} 0$ \\
\hline 13054 & Aug 2, 2014 & 13.00364 & +107.7 & $6{ }^{\prime \prime} 4 \times 1{ }^{\prime \prime} 8$ \\
\hline 13054 & Sep 28, 2014 & 13.03182 & +162.1 & $6 !^{\prime \prime} 4 \times 11^{\prime \prime} 8$ \\
\hline $13395^{f}$ & Dec 16, 2014 & 13.07088 & -113.2 & $6{ }^{\prime \prime} 4 \times 1^{\prime \prime} 8$ \\
\hline 13395 & Jan 16, 2015 & 13.08620 & -85.7 & $6 .^{\prime \prime} 4 \times 22^{\prime \prime} 0$ \\
\hline 13395 & Mar 13, 2015 & 13.11389 & -31.8 & $6 !^{\prime \prime} 4 \times 11^{\prime \prime} 8$ \\
\hline
\end{tabular}

$a$ Phase, $\phi$, refers to $\mathrm{JD}=\mathrm{JD}_{o}+2022.7(\phi-11)$, where $\mathrm{JD}_{o}=2,452,819.2$, corresponding to when the narrow component of HeI $\lambda 6678$ disappears (Groh \& Damineli 2004; Damineli et al. 2008a). Note that periastron is thought to occur about ten days later in the 5.54-year cycle based upon modeling of observations of He II $4686 \AA$ (Teodoro et al. 2016).

${ }^{b}$ Map sampling during the first two visits were $0{ }^{\prime \prime} 1$ intervals perpendicular to the STIS $52^{\prime \prime} \times 0$.' 1 aperture. All other mappings were accomplished at sampling with 0 .' 05 intervals.

${ }^{c}$ Extent of the mapped regions was limited by the 96 minute $H S T$ orbit since all observations were accomplished in continuous viewing orbital opportunities.

${ }^{c}$ All observations discussed in this article were performed with the STIS grating, G430M, centered at $4706 \AA$.

$e$ Observations during the first three visits were done with non-CVZ orbits and hence were constrained to 20 spectra per orbit in order to fit within the HST orbital observing window.

$f$ Observations for the December 2014 were broken into two normal $H S T$ orbits due to a guide star failure during the planned single CVZ orbit scheduled for late November 2014.

excitation of its fossil winds were returning to the highionization state as demonstrated by seeing-limited, groundbased observations done in the same time period (Richardson et al. 2015). Additional mappings (see Table 1) were accomplished through guest investigator programs (Corcoran 2011; Gull 2011, 2012, 2013). Variations in sampling frequency ( 0.1 versus $\left.0 .{ }^{\prime \prime} 05\right)$ and mapped area $\left(1^{\prime \prime}\right.$ versus $\left.2^{\prime \prime}\right)$ demonstrated that optimal sampling is one-half the width of the STIS aperture, 0. . 05 , and that the size of the fossil wind structure required a minimum mapped area of 1 .' 8 in diameter.

When possible, as $\eta$ Car lies in the direction of the HST orbital pole, we requested continuously viewing zone (CVZ) orbits. This allowed us to double the number of acquired spectra per orbit. A full map at each grating setting was accomplished within an individual orbit without visibility interruption by Earth passage. Most visits were accomplished with CVZ orbits. Exceptions were the first three visits done in June 2009, December 2009 and October 2010, plus a replacement visit in December 2014 due to loss of a guide star during the planned November 2014 visit. The November/December 2014 visit was very critical as we needed information on the early recovery structures after the 2014.6 
periastron event. The CCD readout was limited to 128 rows $\left(6{ }^{\prime \prime} 3\right)$ to minimize readout overheads.

Orientation of the $52^{\prime \prime} \times 0^{\prime \prime} .1$ aperture was limited by constraints imposed by HST solar panel orientation, so the PA could not be specified. All mappings were resampled to a common right ascension/declination grid centered on the position of $\eta$ Car.

Earlier ground-based (Richardson et al. 2015), $H S T / S T I S$ observations (Gull et al. 2009) and the 3-D models of the interacting winds (Madura 2010; Gull et al. 2011; Madura 2012; Madura et al. 2013) demonstrated that structural changes were slow across the long, high-ionization state (apastron) when the binary members are furthest apart and moving slowly. HST/STIS visits were scheduled at one year intervals from 2010 to 2013.

Visits were much more frequent before, during and after the periastron event. Across the short, low-ionization state (periastron), the stars approach to within a few AU, moving at velocities relative to each other that approach $v_{\infty, A}$ (Nielsen et al. 2007). From the 3-D models, we selected critical phases where structures were expected to change in ionization, which determined critical times between February 2014 and March 2015 when observations were needed (see Table 1).

The timing of these observations was selected based upon the known changes from high-ionization to lowionization state. Damineli et al. (2008a) and Damineli et al. (2008b) reference $\phi=0$ to the disappearance of the narrow, emission component for He I $6678 \AA$. The present observations sample the high-ionization state at multiple intervals, $\phi=12.163$ through $12.977,13.071$ through 13.114 , but only twice during the low-ionization state, $\phi=13.004$ and 12.032 . While the drop to low-ionization state is well identified, observations of the recovery from low- to high-ionization are very limited during past periastron passages. The most recent recovery was not covered from the ground due to limited night-time visibility. Hopefully substantial coverage will be possible during the recovery phase of the 2020.1 periastron passage as it is well-placed in southern winter.

Data reduction is described in Appendix A.

\section{STRUCTURAL CHANGES WITH ORBITAL PHASE}

Here we describe changes in structures revealed in the light of [Fe III] and [Fe II] from $\phi=12.084$ to 13.114 , a span of 5.7 years extending from early recovery of the low-ionization state across a high-ionization state to nearly full recovery after the next low-ionization state associated August 2014 periastron passage. These changes are predominantly caused by two effects: 1) changes in structures due to photoionization and 2) radial expansion of the wind structures with time.

For the convenience of the reader, $\eta$ Car is at a distance of 2300 pc (Walborn 2012). An angular dimension of $0^{\prime \prime} 1$ translates to $230 \mathrm{AU}$. Over the 5.54-year orbital period, 500 $\mathrm{km} \mathrm{s}^{-1}$ translates to an spatial motion of $516 \mathrm{AU}$ or $0 .{ }^{\prime \prime} 2$ on the sky plane.

We first describe the major changes from apastron to periastron in Section 3.1. A global view with highlights is presented in Section B along with full mosaics of the [Fe III]
(Fig. B1 and the [Fe II] (Fig. B2) iso-velocity images. A brief description of long term changes since 1985 is described in Section 3.2. Changing structures are described in Section 3.3 with focus on the slowly expanding structures, including the Weigelt objects (Section 3.3.1), the rapidly approaching structures (Section 3.3.2) and the rapidly receding structures (Section 3.3.3). A novel means of measuring structural changes is described in Section 3.4. The technique is applied to [Fe III] in Section 3.4.2 and to [Fe II] in Section 3.4.1.

\subsection{The extreme changes from apastron to periastron}

The most noticeable contrast in emission structures revealed by $[\mathrm{Fe} \mathrm{III]} \mathrm{and} \mathrm{[Fe} \mathrm{II]} \mathrm{is} \mathrm{between} \mathrm{apastron,} \mathrm{when} \mathrm{the} \mathrm{two}$ stars are separated by the orbital major axis, $\approx 30 \mathrm{AU}$, and periastron, when the separation drops to $\approx 1.5$ AU (Fig. 1). The 3-D hydrodynamic models (Okazaki et al. 2008; Pittard \& Corcoran 2002; Parkin et al. 2009, 2011; Madura 2012; Madura et al. 2013; Clementel et al. 2015a,b) demonstrate that near apastron, the cavity carved by $\eta$ Car B out of the primary wind is open and nearly parabolic, allowing FUV, originating from $\eta$ Car B, to escape the current wind-forming cavity $^{5}$.

As the system approaches periastron, the distance between the two stars decreases, and, since the distance of the wind-wind shock'wall' from $\eta$ Car B scales with the stellar separation, the shock boundary moves closer to $\eta$ Car B. By periastron, $\eta$ Car B's orbital velocity becomes comparable to the primary wind terminal velocity. The cavity becomes greatly distorted with the leading edge of the bowshock penetrating deeply into the primary wind and with the trailing edge far behind the secondary wind. Rapidly, FUV radiation from $\eta$ Car B is blocked by the slow-moving primary wind. Hydrogen in the primary wind absorbs the FUV, converting it to radiation with energies less than $13.6 \mathrm{eV}$ and an optically thick Lyman $\alpha$ radiation field $(10.4 \mathrm{eV})$. The high-ionization state is no longer supported external to the very shrunken wind-wind cavity. The mid-ultraviolet radiation, $\approx 10 \mathrm{eV}$ to $5 \mathrm{eV}$ (MUV), which comes from both the primary and the converted FUV from the secondary, escapes the wind-wind cavity. Throughout the fossil wind structures, iron formerly as $\mathrm{Fe}^{++}$drops to $\mathrm{Fe}^{+}$and many [Fe III] structures reappear as [Fe II] structures.

These changes are driven by UV radiation, absorption of the UV radiation, expanding structures and densities of the excited gas. Iron is ionized to $\mathrm{Fe}^{+}$by MUV exceeding $7.9 \mathrm{eV}$ and further ionized to $\mathrm{Fe}^{++}$by FUV above $16.2 \mathrm{eV}$. Thermal excitation populates these ions to the upper state of the transitions in question, which occur at $2.7 \mathrm{eV}$ with critical

${ }^{5}$ While the predominant source of FUV radiation is thought to be $\eta$ Car B, the colliding winds, the source of X-rays which reach a shallow minimum across periastron, may also contribute. However we would expect the FUV radiation from the wind-wind collision zone to be least near apastron when the two stars are most distant and to increase with approaching periastron. A complication comes up with evidence that as periastron approaches, the winds reach a stalling point (Clementel et al. 2015b). Additional modeling is necessary to determine the contribution by the colliding winds. 

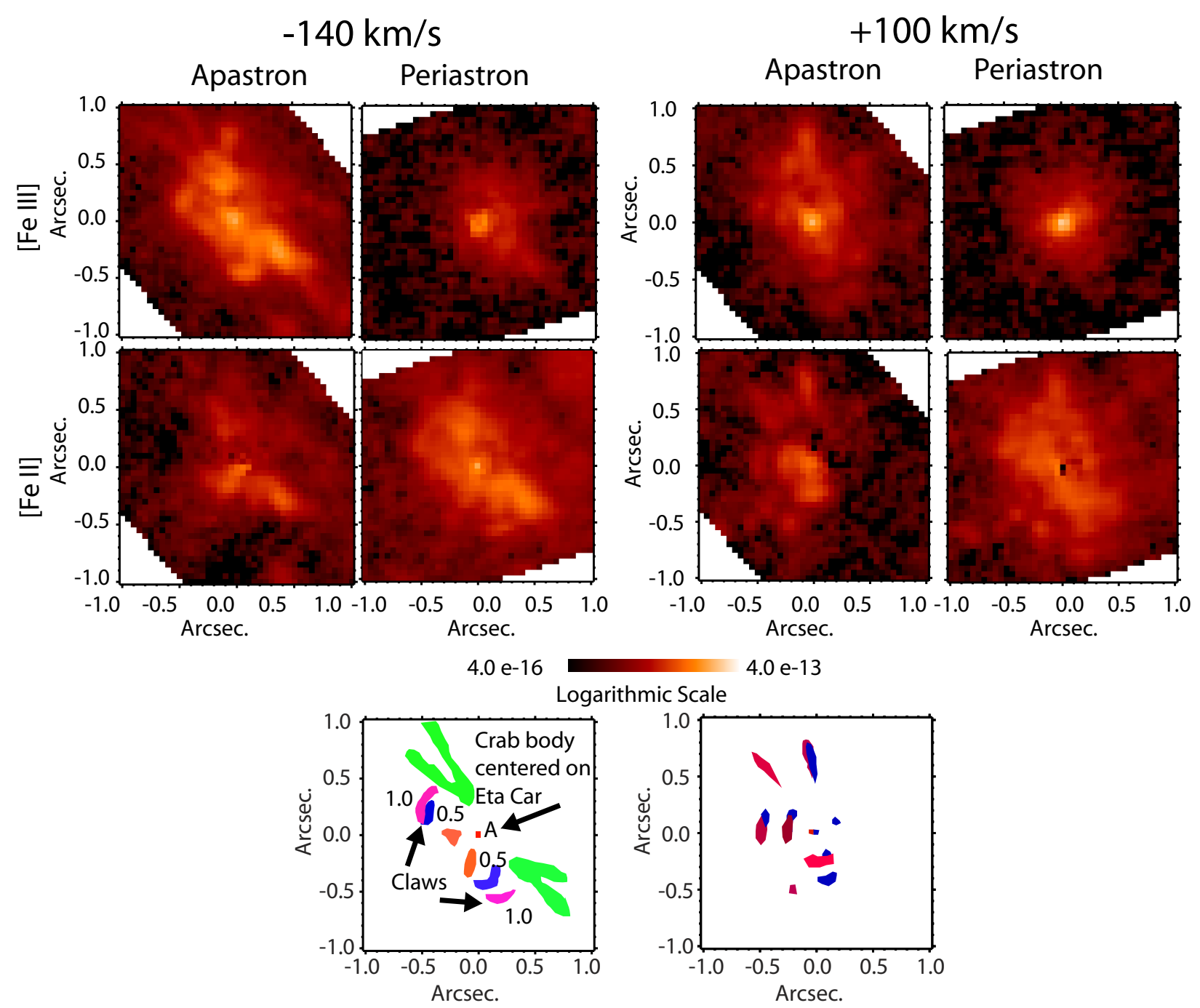

4.0 e-13

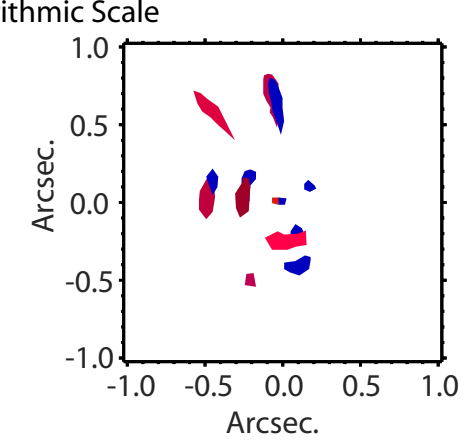

Figure 1. Changes between apastron $(\phi=12.516)$ and periastron $(\phi=13.004)$. Top Left: Comparison of $-140 \mathrm{~km} \mathrm{~s}{ }^{-1}$ images Structures seen in [Fe III] at apastron (upper left) appear in [Fe II] at periastron (lower right), but having expanded radially from $\eta$ Car over the 2.77 year interval. Top Right: Comparison of $+100 \mathrm{~km} \mathrm{~s}^{-1}$ images between apastron and periastron. Again structures seen in $[\mathrm{Fe}$ III] at apastron are seen in [Fe II] at periastron, having expanded over the time interval. Bottom Left: Sketch depicting selected $-140 \mathrm{~km} \mathrm{~s}^{-1}$ filaments seen in [Fe III] at apastron and in [Fe II] at periastron. Arcuate structures (blue) seen in [Fe III] expanded outwardly and appear in [Fe II] (pink) at periastron. New arcuate structures (orange) are seen in [Fe III] at apastron and in [Fe II] at periastron. Bifurcated structures (green) seen in [Fe III] at apastron are not obvious in [Fe II] at periastron. Bottom Right: Sketch of $+100 \mathrm{~km} \mathrm{~s}^{-1}$ structures seen in [Fe III] (blue) and in [Fe II] (red) at apastron. Most structures show interior [Fe III] and exterior [Fe II] components, consistent with photoionization from the central source. Expansion in [Fe III] across the high-ionization state is consistent with 450 to $470 \mathrm{~km} \mathrm{~s}^{-1}$, close to the $v_{\infty, A}=420 \mathrm{~km} \mathrm{~s}^{-1}$ derived by Groh et al. (2012) and the $470 \mathrm{~km} \mathrm{~s}^{-1}$ expansion velocity of [Fe II] structures as measured by Teodoro et al. (2013). However, shifts in illumination due to the motion of the secondary (FUV source) star, intervening structures that first absorb FUV radiation, then break open, and expansion of the shells lead to rotational shifts of the [Fe III] features: i.e. different portions of the shells emit [Fe III] as the orbit progresses. By contrast the arcuate features emitting [Fe II] appear to move outward radially (note: all images in this figure have continuum subtraction).

densities for the transitions of interest: [Fe III] $4659.35 \AA$ : $\mathrm{n}_{e}=10^{7} \mathrm{~cm}^{-3}$ and [Fe II] 4815.88̊: $\mathrm{n}_{e}=2 \times 10^{6} \mathrm{~cm}^{-3}$.

The extremes of the high- and low-ionization states are compared for $-140 \mathrm{~km} \mathrm{~s}^{-1}$ and $+100 \mathrm{~km} \mathrm{~s}^{-1}$ velocities in Fig. 1. The [Fe III] structures, visible at apastron, disappear at periastron only to reappear as expanded, more diffuse, $[\mathrm{Fe}$ II] structures. The periastron [Fe II] structures are enlarged relative to the apastron [Fe III] structures due to radial expansion during the 2.7-year time interval between $\phi=12.516$ and 13.004 .
This effect is most noticeable in the $-140 \mathrm{~km} \mathrm{~s}^{-1}$ images in Fig. 1. As drawn in the cartoon (bottom left) two [Fe III] arcs, visible at apastron (labeled 0.5), shift outward and change to [Fe II] arcs, visible at periastron (labeled 1.0). Two pairs of very extended [Fe III] structures (green in the left cartoon) are not seen in [Fe II] other than structures closest to $\eta$ Car. In general, periastron-visible [Fe II] structures spatially project to the outside of the apastron-visible [Fe III] structures.

The contrast is less for $+100 \mathrm{~km} \mathrm{~s}^{-1}$ structures dis- 
played in Fig. 1. Less [Fe III] emission is apparent at apastron as these structures are on the far side of $\eta$ Car where little FUV radiation can penetrate. Close to $\eta$ Car are several arcuate [Fe III] structures that form a nearly complete 0. . 6 diameter ring, plus a faint streak that projects directly north (blue in the cartoon in Fig. 1, right). The [Fe II] structures at apastron clearly define two sets of arcs, plus a pair of streaks, one associable with the [Fe III] streak to the north and a second to the northeast. The [Fe II] emission at periastron reveals many additional arcs/clumps at distances out to nearly 1 .' 0 . These additional arcs are likely structures that, at apastron, are ionized by the FUV radiation but have densities lower than $\mathrm{n}_{e}=10^{7} \mathrm{~cm}^{-3}$, comparable to $\mathrm{n}_{e}=2 \times 10^{6}$ $\mathrm{cm}^{-3}$, the critical density for the [Fe II] $4815.88 \AA$ emission. Hence they are not seen in [Fe III] at apastron, but in [Fe II] at periastron.

\subsection{Long term changes since 1985}

In the time since Weigelt \& Ebersberger (1986) and Hofmann \& Weigelt (1988) resolved $\eta$ Car plus three point-like sources within 0 .' 3 via ground-based speckle interferometry (Fig. 2.a), the apparent brightness of the central sources has increased by nearly a factor of three at visible wavelengths, part of a trend since 1940 (Davidson et al. 1999; FernándezLajús et al. 2009). A decade later Weigelt et al. (1995) via the HST/FOC confirmed these stellar-like sources persisted (Fig. 2.b). Davidson et al. (1995b) obtained observations with $H S T / F O S$ that demonstrated the spectrum of Weigelt A is stellar-like with a hot, dense wind, while objects B, C and $\mathrm{D}$ are ejecta with many forbidden lines. Proper motion studies by Weigelt et al. (1995) and Smith et al. (2004) suggested these objects were ejected between 1880 and 1930 while Dorland et al. (2004) found ejection between 1900 and 1950. More recently Weigelt \& Kraus (2012) found a proper motion of $\mathrm{C}$ and $\mathrm{D}$ of 2 mas $\mathrm{yr}^{-1}$ consistent with ejection between 1860 and 1900 .

Long aperture spectroscopy with HST/STIS from 1998 to 2004 showed that emission line fluxes of Weigelt D did not increase proportionately with the flux of $\eta$ Car (Hillier \& Allen 1992; Hillier et al. 2001; Gull et al. 2009; Mehner et al. 2010). The apparent brightening appears to be due to changes in obscuration between $\eta$ Car and the observer, but not in the direction from Weigelt $\mathrm{D}$ to the observer. Weigelt et al. (1995) suggested an elongated obscuring structure to be a circumstellar disk in front of $\eta$ Car at PA $50^{\circ}$ which is orthogonal to the symmetry axis of the Homunculus. To study this occulting structure, Falcke et al. (1996) carried out speckle-masking, imaging polarimetry in $\mathrm{H} \alpha$. They obtained images (see their Fig. 2b) that show a northeast to southwest bar in polarized light about 0. . 4 long oriented at $\mathrm{PA} \sim 45^{\circ}$. This observed northeast to southwest bar was interpreted to be an equatorial disk. The polarized bar has approximately the same length and PA as that of the extended emission structures discovered by Gull et al. (2011).

Comparisons are presented in Fig. 2 of images recorded by speckle masking in the 1980s (Fig. 2.a), by $H S T / F O C$ in the mid-1990s (Fig. 2.b) and by $H S T / S T I S$ mappings in 2011 (Fig. 2.c-e). Integration of the STIS-mapped data across the complete spectrum recorded with the G750M grating centered at $5734 \AA$ (Fig. 2.c) do not readily show the Weigelt objects. The dominant structure appears to be a diffuse structure in the diffraction ring region, plus a streak along the aperture when placed directly on $\eta$ Car. Constraining the velocity of the image to $-40 \mathrm{~km} \mathrm{~s}^{-1}$ with width of $40 \mathrm{~km} \mathrm{~s}^{-1}$ (comparable to the instrumental resolving power, $\mathrm{R}=\lambda / \delta \lambda=8000$ ), centered at $[\mathrm{N} \mathrm{II}] 5756.19 \AA$. Fig. 2.d reveals a hook-shaped structure offset to the north and west from $\eta$ Car. When continuum is subtracted, the hook structure becomes quite apparent (Fig. 2.e). The two brightest peaks in Fig. 2.e correspond closely to the positions

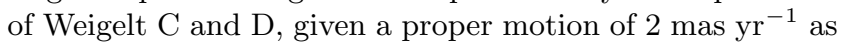
measured by Weigelt \& Kraus (2012). For the reader's benefit, a cartoon (Fig. 2.f) illustrates the positions of Weigelt C and D relative to $\eta$ Car at the time of the STIS observation. However, as noted by Teodoro et al. (in prep) the emission line shapes of Weigelt $\mathrm{C}$ and $\mathrm{D}$ change with orbital phase. Weigelt $\mathrm{B}$ is not detected in any mappings recorded between 2009.4 and 2014.3, likely because it has dissipated through heating, photoionization and wind ablation effects. Furthermore, the structure of Weigelt $\mathrm{C}$ and $\mathrm{D}$ are slowly evolving and eventually will dissipate. Changes in structure due to these effects must also influence proper motion studies.

\subsection{Detailed comparisons of [Fe II] and [Fe III] over the 5.54-year cycle}

Figs. 3 through 7 display selected velocity slices comparing the differences and changes in [Fe III] and [Fe II] in greater detail. All velocity slices utilize a log scale of the flux ranging from $4 \times 10^{-16}$ to $4 \times 10^{-13} \mathrm{erg} \mathrm{cm}^{-2} \mathrm{sec}^{-1}$, which covers the range in flux seen in both [Fe III] and [Fe II] in all velocity frames. Each figure has four columns:

The left two columns display [Fe II] and [Fe III] for the seven observations spanning from early recovery of the high-ionization state $(\phi=12.084)$ to late stages before periastron $(\phi=12.922)$. Since the orbit is very eccentric, the hot secondary spends most of the orbit nearly $30 \mathrm{AU}$ from the primary; its distance and orbital angle change very slowly, so the photo-ionized structures evolve more slowly. The dominant changes are expansions of the fossil winds and apparent shifts in illumination of slow-moving, irregular structures which we label the light house effect (Fig. 9).

The right two columns display [Fe II] and [Fe III] for seven observations spanning from the late high-ionization state $(\phi=12.922)$ across the periastron-induced lowionization state to the early high-ionization state $(\phi=$ 13.114). Note that the maps from $\phi=12.922$ are displayed at both the bottom of the high-ionization state (left two columns) and top of the low-ionization state (right two columns) to enable visual comparisons. Changes are rapid across the low-ionization state. Within the resources allocated, we attempted to sample as frequently as possible, given the CVZ opportunity once every 57 days. While noticeable changes occur between $\phi=12.922$ and 12.977 , the [Fe III] really drops between $\phi=12.977$ and 13.004. Welldefined structures disappear leaving a diffuse haze at every velocity, most noticeably at $-40 \mathrm{~km} \mathrm{~s}^{-1}$ (Fig. 3) for both $\phi=13.004$ and 13.032. By $\phi=13.071$, [Fe III] structures reappear in early recovery of the high-ionization state. Maps at $\phi=13.086$ and 13.114 show continued recovery to the high-ionization state that can be compared to the maps at 12.084 and 12.163 , mapped at half of the optimal sampling frequency. 


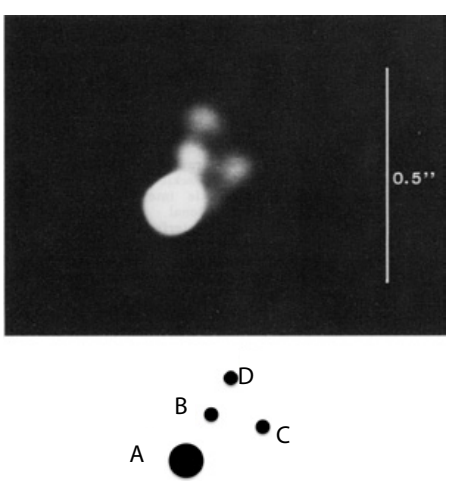

a

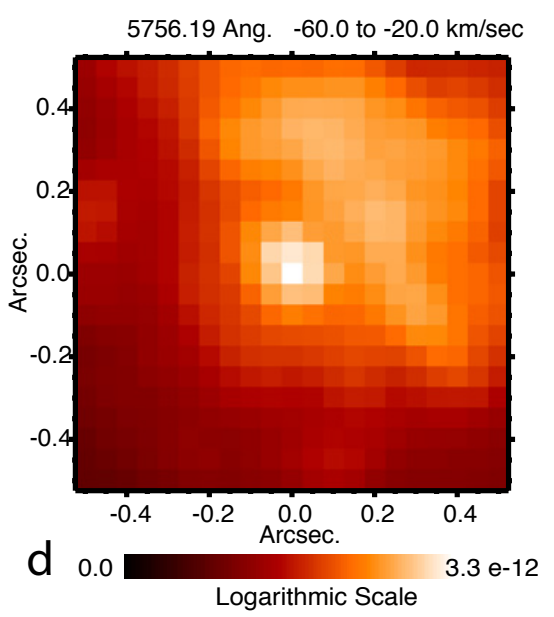

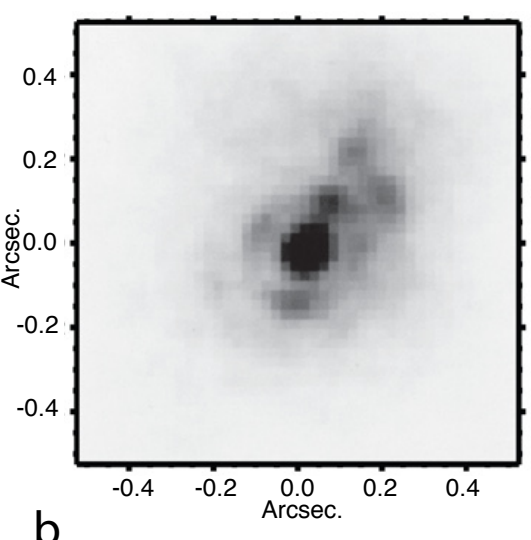

5756.19 Ang. -60.0 to $-20.0 \mathrm{~km} / \mathrm{sec}$

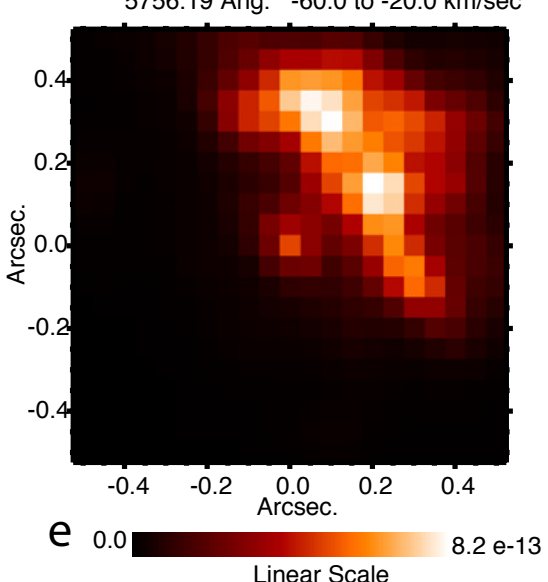

5734 Ang. -12000 to $10000 \mathrm{~km} / \mathrm{sec}$

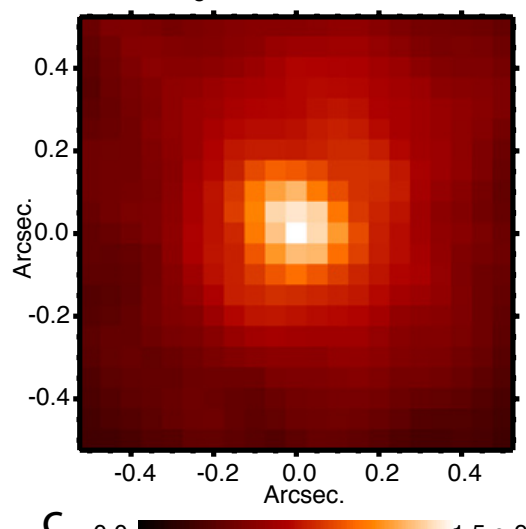

Logarithmic Scale

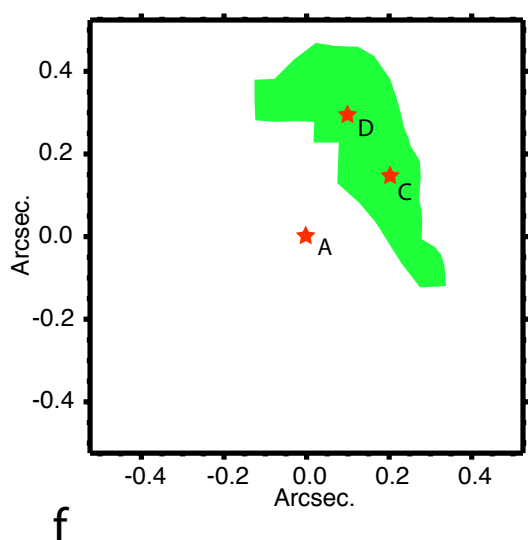

Figure 2. Comparison of the current structures and how they relate to the original Weigelt objects. a: Speckle image recorded in 1985 as published by Hofmann \& Weigelt (1988). Spectral response was from 8300 to $8800 \AA$. Note the sketch at bottom showing four point-like objects. A is $\eta$ Car. B, C and D are very bright emission clumps known as the Weigelt blobs. b: $H S T / F O C$ image as published by Weigelt et al. (1995). The spectral bandpass was defined by the FOC F550M filter and S20 photocathode response. c: $H S T / S T I S$ image integrated from 5500 to $\mathbf{5 9 2 0 \AA}$, the full range of the G750M grating recorded on November 2011 (apastron, $\phi=12.516$ ). Little or no evidence of Weigelt objects B, C or D is obvious in this broadband image. d: HST/STIS image from the same mapping with the bandwidth constrained to $40 \mathrm{~km} \mathrm{~s}^{-1}$ centered at $-40 \mathrm{~km} \mathrm{~s}$ for [N II] $5756.19 \AA$. The bright central component is $\eta$ Car. The extended, hook-shaped structure to the northwest is in the vicinity of Weigelt C and D. Continuum has NOT been subtracted from either frames c or d. e: The continuum-subtracted HST/STIS image with $40 \mathrm{~km} \mathrm{~s}{ }^{-1}$ bandwidth centered at $-40 \mathrm{~km} \mathrm{~s}^{-1}$ for [N II] 5756.19 $\AA$. Faint [N II] emission is centered at coordinates 0,0 which is the position of $\eta$ Car. Weigelt B is not visible at any of the bright emission lines since these mappings began in 2009. f: Sketch depicting the positions of $\eta$ Car (A), Weigelt $\mathbf{C}$ and $\mathbf{D}$, plus the hook-shaped nebular structure that extends around $\mathbf{C}$ and $\mathbf{D}$. As noted by Teodoro et al. (in prep, 2016) the peak nebular emission positions in the current epoch do not correspond to previously measured positions of $\mathrm{B}, \mathrm{C}$ and $\mathrm{D}$ due to proper motion, changes in photoionization, illumination and possible ablation. The change from the three clumps of emission, detected in 1985 and 1995, to the more diffuse structures by 2011 suggests that all three Weigelt objects are slowly evolving and dispersing. Note that these images are dimensioned $1^{\prime \prime} \times 1^{\prime \prime}$. All other images in this article are $2^{\prime \prime} \times 2^{\prime \prime}$. Images presented in c.-e. have been logarithmically scaled as shown in each color wedge to maximize displayed range. Images a and b are used with permission of the authors.

\subsubsection{The slowly moving structures}

The strongest forbidden emissions originate in Weigelt $\mathrm{C}$ and $\mathrm{D}$, plus associated, fainter structures in the velocity interval from -80 to $0 \mathrm{~km} \mathrm{~s}^{-1}$ (see Fig. 3 for the $-40 \mathrm{~km} \mathrm{~s}^{-1}$ isovelocity images with $40 \mathrm{~km} \mathrm{~s}^{-1}$ width). In general, Weigelt $\mathrm{C}$ is slightly brighter than Weigelt $\mathrm{D}$, but small shifts in relative brightness and apparent position occur across the cycle.

The under-sampled maps at $\phi=12.084$ and 12.163 (Fig. 3) are very similar to the optimally sampled maps at $\phi=$
13.086 and 13.114 , one orbital period later, which indicates that the low-velocity structures have not evolved much in one 5.54-year period, that the UV sources of ionization for $\mathrm{Fe}^{++}(16.2 \mathrm{eV})$ and $\mathrm{Fe}^{+}(7.9 \mathrm{eV})$ have not changed significantly nor have the densities of the current fossil structures changed much from the previous fossil structures at comparable phases one cycle earlier.

The structures of greater importance are the much fainter outer structures that appear and disappear across the cycle: 
(i) Two arcuate segments of [Fe III $]$ appear by $\phi=12.315$ : one located $0 . .4$ at $\mathrm{PA}=80^{\circ}$ and the other located $0 . \prime 4$ at $\mathrm{PA}=190^{\circ}$ from $\eta$ Car(see structure indicated by arrows for $\phi=12.315$ in Fig. 3). These arcuate segments, which we name 'claws' (as in crab claws), reappear in other iso-velocity slices. In following sections, the claws are followed as they change with phase.

(ii) These [Fe III] claws expand outwardly with advancing phase but begin to fade by $\phi=12.922$ while brightening in [Fe II].

(iii) A second, fainter pair of [Fe III] claws appears at about the same PA, 0.'2 from $\eta$ Car but are not as obvious across the high-ionization state from $\phi=12.516$ onward. They reappear as [Fe II] claws across perastron $(\phi=13.004$ and 13.032) about $0 . \prime 4$ from $\eta$ Car at PA $=60$ and $200^{\circ}$.

(iv) At $\phi=13.004$ and 13.032 the outer claws, now visible in [Fe II], have expanded to $\approx 0$.' 6 , are more extensive and are joined by two internal pairs at $\approx 0 . \prime 4$ and $0 . \prime 2$. The three pairs of arcuate segments indicate repeated formation of shells each cycle.

(v) By $\phi=13.071$, a single [Fe III $]$ claw at $0^{\prime \prime} 6, \mathrm{PA}=80^{\circ}$ is again visible, but the claw at $0 .{ }^{\prime \prime}, \mathrm{PA}=190^{\circ}$ has not reappeared.

(vi) No additional claws are visible at $\phi=13.114$, similar to no claws being visible at $\phi=12.163$.

\subsubsection{The blue-shifted structures}

Considerable variations are identifiable in the blue-shifted structures. Figs. 4 and 5 reproduce iso-velocity images for -140 and $-260 \mathrm{~km} \mathrm{~s}^{-1}$ as examples of these many variations. Several cyclic changes are obvious in the blue-shifted frames:

(i) $[\mathrm{Fe} \mathrm{II}]$ is most extensive across the low-ionization state ( $\phi=13.004$ to 13.071 ), but fades across the high-ionization state with minimal emission near apastron. Little or no [Fe II] is seen at the position of $\eta$ Car.

(ii) $[\mathrm{Fe}$ III $]$ is extensive across the high-ionization state but nearly disappears across the low-ionization state. The peak flux usually at the position of $\eta$ Car.

(iii) With increasing blue-shift, decreasing [Fe II] emission structures are present, other than across the low-ionization state when [Fe III] emission fades and is replaced by [Fe II] emission.

(iv) [Fe III $]$ emission across the high-ionization state develops into a crab-shaped structure, rotated to $-45^{\circ}$, extending from the northeast to the southwest centered about $\eta$ Car. With the two claws that appear at $\mathrm{PA}=80^{\circ}, 0^{\prime \prime} .4$ and $\mathrm{PA}=190^{\circ}, 0^{\prime \prime} .4$, the two extended structures $\mathrm{PA}=0^{\circ}$ positioned 0.5 and $\mathrm{PA}=315^{\circ} 0^{\prime \prime} .5$, plus the head-like $\eta$ Car, the structure resembles a crab in Fig. 4 at $\phi=12.315$.. Appropriately the claws expand outward with orbital phase across the high-ionization state. This structure was noted by Gull et al. (2009) in the broader velocity interval from -200 to $-400 \mathrm{~km} \mathrm{~s}^{-1}$ interval.

(v) Many [Fe II] emission structures lie radially just beyond [Fe III] structures relative to $\eta$ Car.

(vi) Very little blue-shifted structure is seen to the northwest of $\eta$ Car.

(vii) As noted with the low velocity frames, the maps recorded at $\phi=12.084$ and 12.163 , allowing for under sampling in the first observations, are remarkably similar to the maps recorded at $\phi=13.086$ and 13.114 .

Specific variations are:

(i) The $[\mathrm{Fe}$ III $]$ structure, that appears crab-like complete with body and large claws in Fig. 4 at $\phi=12.315$, persists through $\phi=12.977$. Most notably the claws expand radially outward with progressing phase. Measures of the [Fe III] expansion proved to be inconsistent when measured at different phases across the long, high-ionization state. By contrast, measurements of the multiple shells seen in the red-shifted [Fe II] and [Ni II] maps led to a consistent expansion velocity of $470 \mathrm{~km} \mathrm{~s}^{-1}$ (Teodoro et al. 2013). Careful examination of the multiple velocity frames revealed that the structure shifts tangentially as well as radially. Likely the [Fe III] emission originates from spatially different regions as $\eta$ Car B slowly changes illumination angle as seen by the fossil shell -analogous to a scanning beam from a (drifting) lighthouse boat illuminating a distant, moving ship through a shifting fog. Here the fog may be due to thin, compressed primary-wind walls moving in our general direction. Similar, small- and large-scale effects have been noted previously (Smith et al. 2004; Madura \& Groh 2012).

These [Fe III] claws fade as the high-ionization state shifts towards the low-ionization state (Fig. $4, \phi=12.922$ to $12.977)$. Already very faint at $\phi=12.977$, the claws, and all other [Fe III] structures, disappear by $\phi=13.004$. Only a diffuse, faint structure remains to the northwest, offset from core [Fe III] emission at the position of $\eta$ Car. The claw at $\mathrm{PA}=80^{\circ}$ reappears by $\phi=13.071$ and may be joined by a faint, second claw at $\mathrm{PA}=190^{\circ}$. Both are about $0^{\prime \prime} 6$ separated from $\eta$ Car.

The claws are not seen in [Fe II] until $\phi=12.922$. They brighten to a maximum across the low-ionization state. The $\mathrm{PA}=80^{\circ}$ claw disappears by 13.071 but the claw at $\mathrm{PA}=$ $190^{\circ}$ may be present until $\phi=13.086$.

(ii) Shadowing effects become apparent when comparing [Fe II] to [Fe III] frames for each phase. An example is to be seen in Fig. 4 comparing the forbidden line maps recorded at $\phi=12.163,13.071,13.086$ and 13.114 . An apparent cavity is defined between $\mathrm{PA}=40^{\circ}$ and $85^{\circ}$ in [Fe II], but in [Fe III] a crab claw appears at $\mathrm{PA}=80^{\circ}$ at $\phi=13.071$ while the $[\mathrm{Fe}$ II] persists. A spike of [Fe III] emission bounds the [Fe II] edge at $\mathrm{PA}=40^{\circ}$ at $\phi=12.163$ and 13.086. A second spike is seen in $\phi=12.084$ and 12.163 extending to the edge of the $2^{\prime \prime} \times 2^{\prime \prime}$ frame at $\mathrm{PA}=230^{\circ}$, but does not appear one cycle later at $\phi=13.086$ nor 13.114. These spikes are NOT instrumental as they do not conform to a row or column of the maps and are seen at the same PAs even though the HST-defined PA for the STIS aperture changes between visits. A second spike can be seen in [Fe III] at phases $\phi=12.516$ and 12.681 at $\mathrm{PA}=30^{\circ}$. This spike also appears in $[\mathrm{Fe} \mathrm{III}]$ at $\phi=12.977$ along with the spike at $\mathrm{PA}=225^{\circ}$.

These shadows and spikes, seen at $\phi=13.071$ to 13.114 , most likely trace out boundaries in wind-wind structures and fossil structures that pass or block the FUV radiation, thus leading to gaps in [Fe III] high-velocity emission that are filled in by [Fe II] emission. A second, much less obvious, shadow may be to the south of $\eta$ Car, in the direction from $\mathrm{PA}=160$ to $190^{\circ}$. The region between the two shadows, $\mathrm{PA}=$ 90 to $160^{\circ}$, shows up in [Fe II]. 
High State
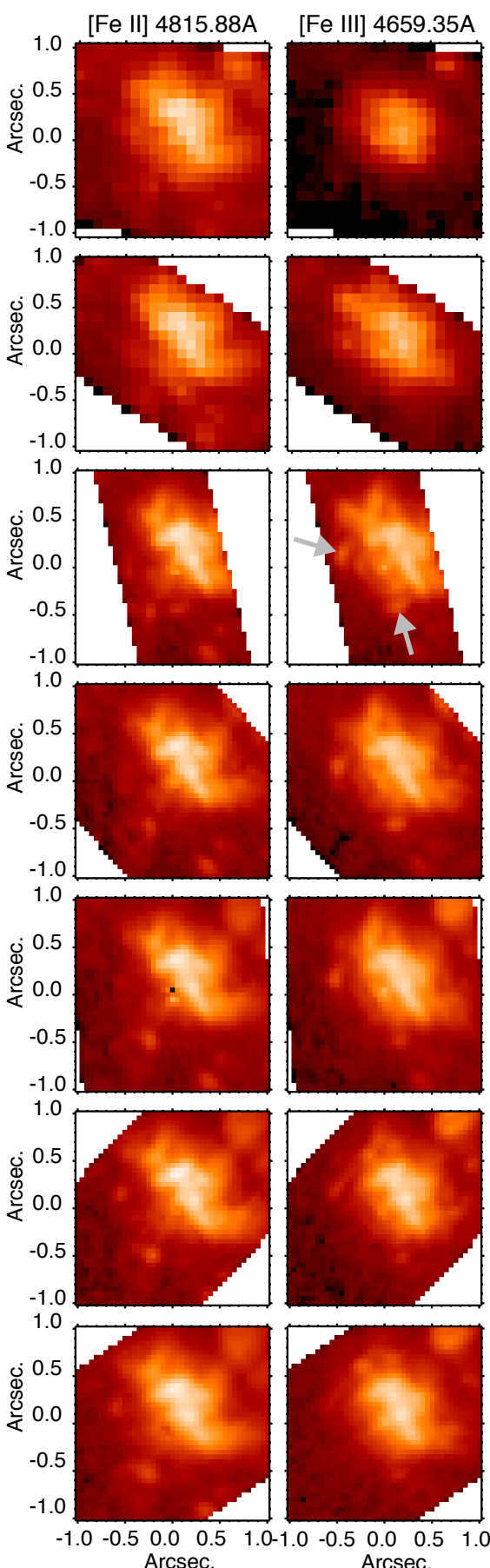

4 e-16 4 e-13

Logarithmic Scale
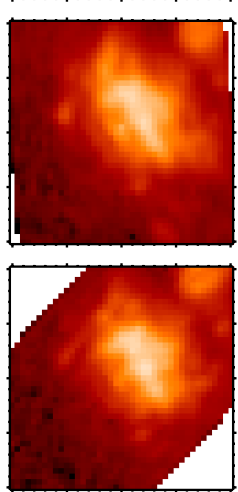

Low State
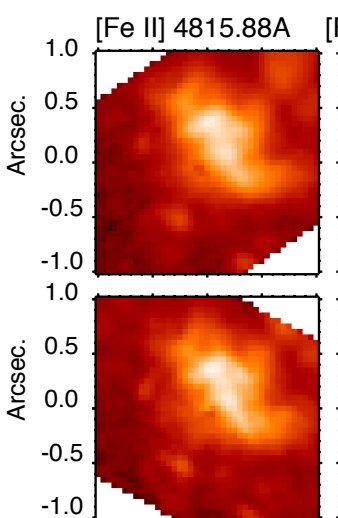

1.0
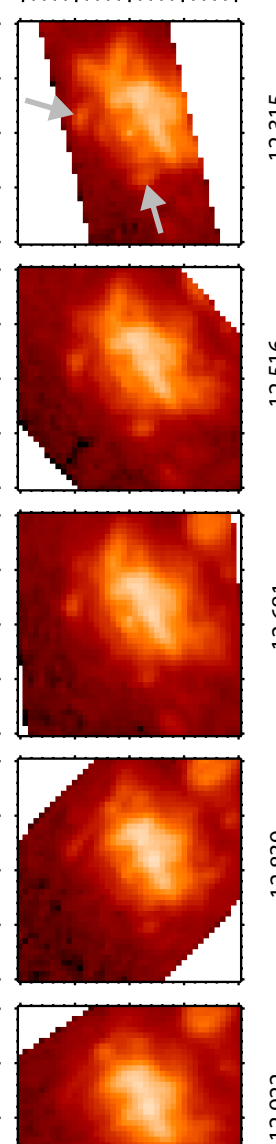

م⿸
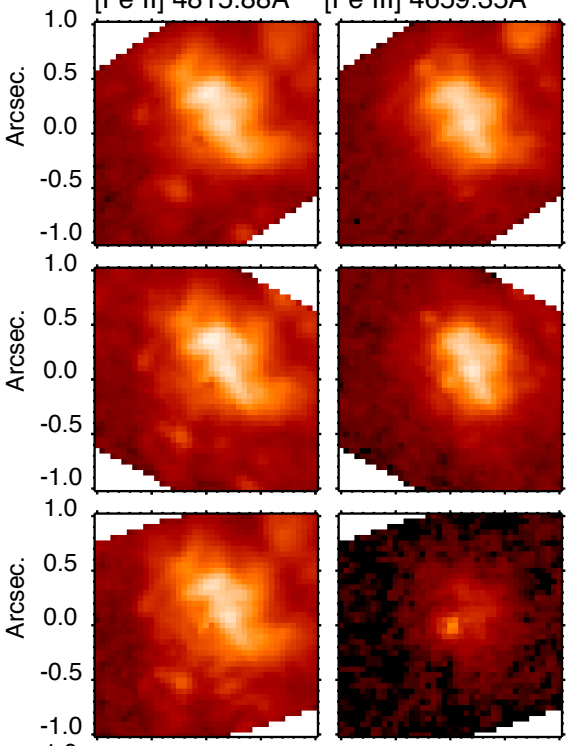

สิ
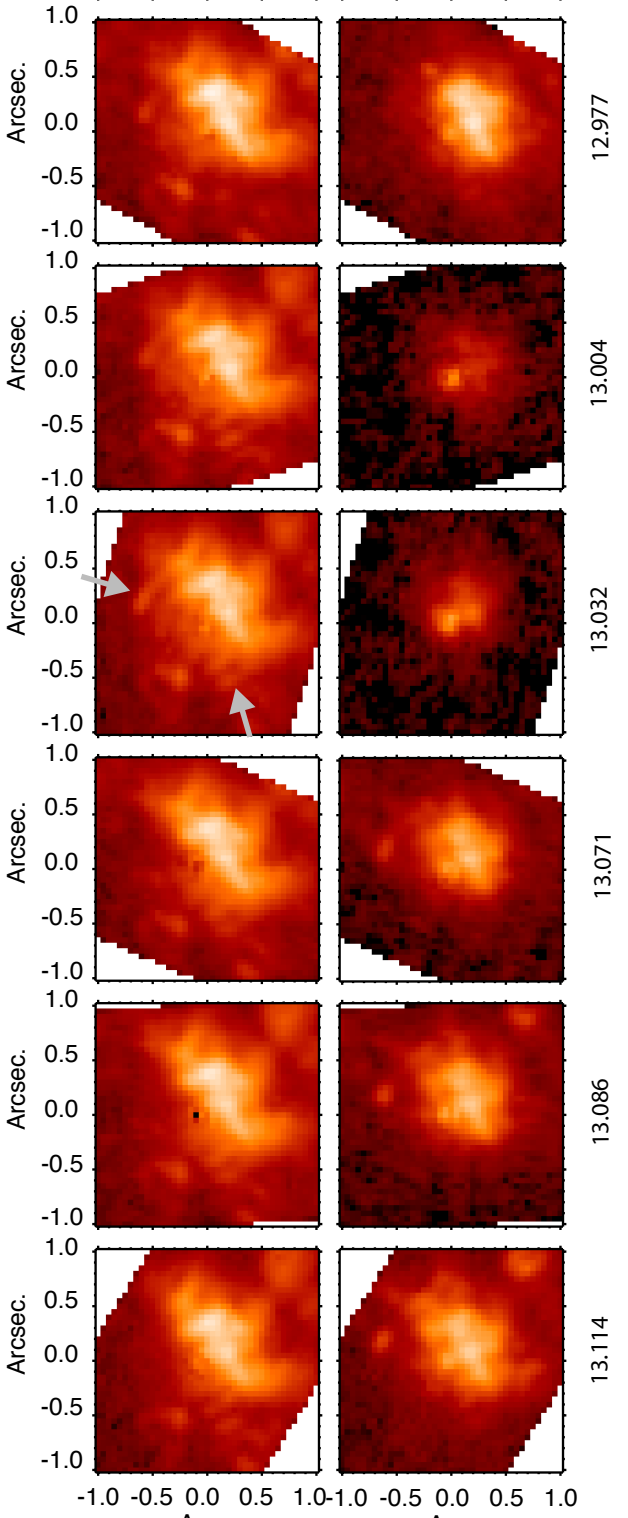

$\underset{\hat{s}}{\grave{n}}$

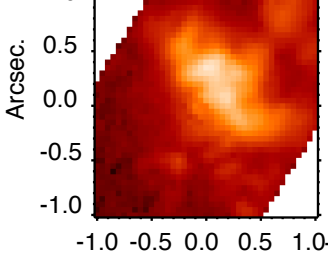

Arcsec.

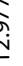

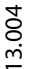

$\stackrel{\sim}{m}$

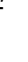

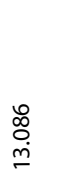

$\stackrel{\circ}{\stackrel{\circ}{\infty}}$

\section{$-40 \mathrm{~km} / \mathrm{s}$}

Figure 3. Changes in spatial structure from 2009.4 to $2014.2:-40 \mathrm{~km} \mathrm{~s}^{-1}$. Left two columns: Iso-velocity slices of [Fe II] and $[\mathrm{Fe}$ III] for the seven mappings across the high-ionization state from phases 12.084 to 12.922 . Right two columns: Iso-velocity slices of [Fe II] and [Fe III] across the low-ionization state from $\phi=12.922$ to 13.114. The Weigelt objects and the hook structure (Fig. 2) change slowly in [Fe II] but nearly disappear in [Fe III] across the low-ionization state. Fainter, outer structures visibly shift outward from $\eta$ Car. Notable are two [Fe III] arcuate segments that appear in [Fe III] at $\phi=12.315$, one 0 .' 4 east (grey arrows, [Fe III] at $\phi=$ 12.315 ), the other $0{ }^{\prime \prime} 4$ south of $\eta$ Car. These arcuate segments are faint at $\phi=12.315$, become more prominent by $\phi=12.516$ and shift radially outward across the high-ionization state. By $\phi=12.922$, these arcs have moved to $0^{\prime \prime} 6$ distance from $\eta$ Car and are fading in [Fe III]. The arcuate structures brighten in [Fe II] (grey arrows, [Fe II], $\phi=13.032$ ) as the system goes through the low-ionization state $(\phi=13.004$ and 13.032) and are joined by two interior pairs. By recovery at $\phi=13.114$, the eastern arc is still visible, but the southern arc is very faint. The $\phi=12.922$ mapping is displayed twice, at the bottom of the high-ionization state columns and the top of the low-ionization state columns, as it is a transitional phase at the end of the high-ionization state and beginning of the low-ionization state. 
High State
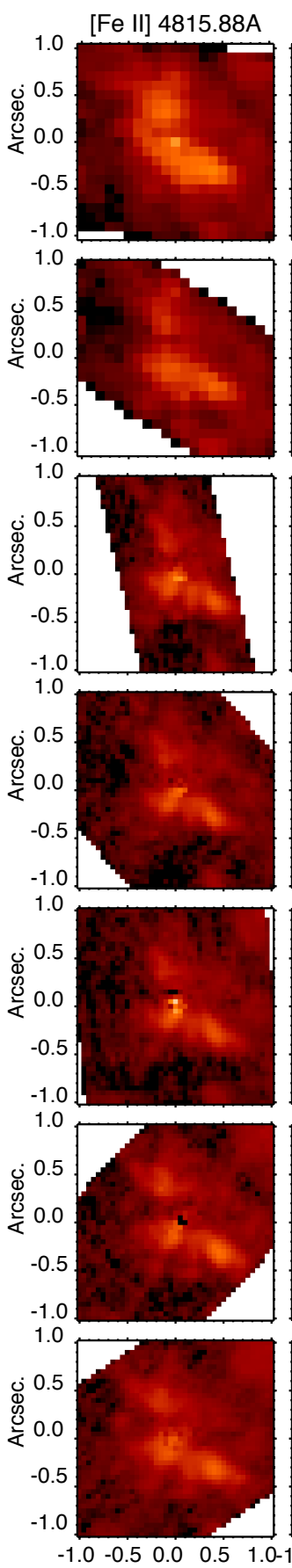

Arcsec.
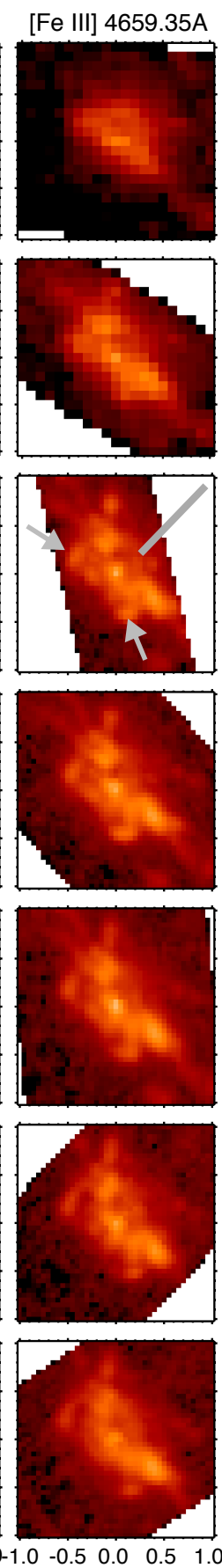

Arcsec.

4 e-16 4 e-13

Logarithmic Scale

Low State

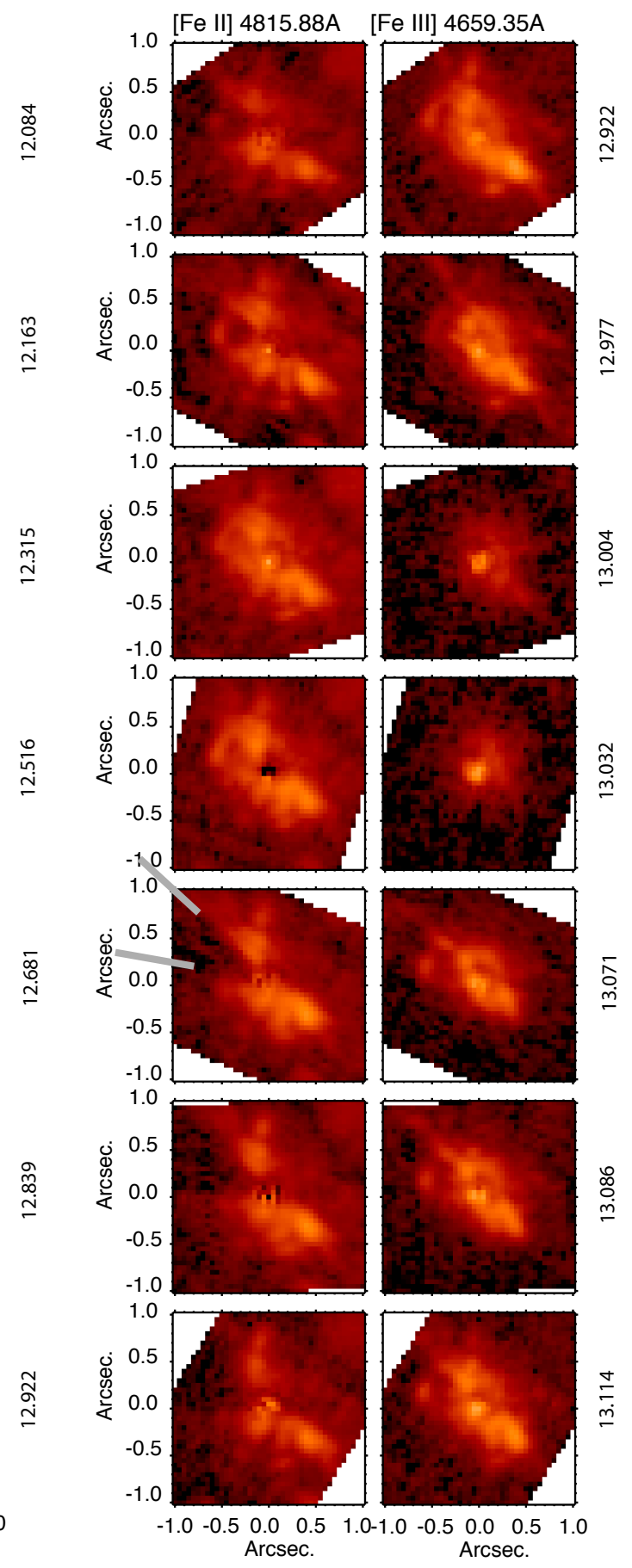

$-140 \mathrm{~km} / \mathrm{s}$

Figure 4. Changes in spatial structure from 2009.4 to $2014.2:-140 \mathrm{~km} \mathrm{~s}^{-1}$. Left: Iso-velocity slices of [Fe II] and [Fe III] for the seven mappings across the high-ionization state from $\phi=12.084$ to 12.922 . Right: Iso-velocity slices across the low-ionization state from $\phi=12.922$ to 13.114 . Two [Fe III] claws (grey arrows, [Fe III], $\phi=12.315$ ) develop 0'” 3 radially separated from $\eta$ Car at $\mathrm{PA}=60$ and $200^{\circ}$ beginning by $\phi=12.315$, radially expand and change in photo-ionization across the cycle. The image of a crab can be imagined, with the body rotated to $-45^{\circ}$ (axis indicated by grey line). A dark cone developed after periastron in [Fe II] (grey lines, $[\mathrm{Fe} \mathrm{II}], \phi=13.071$ ), bordered by an [Fe III] streak. Note that the very bright, slowly moving structures, including Weigelt C and D, seen in low velocity frames (Fig. 3) have disappeared. 
High State
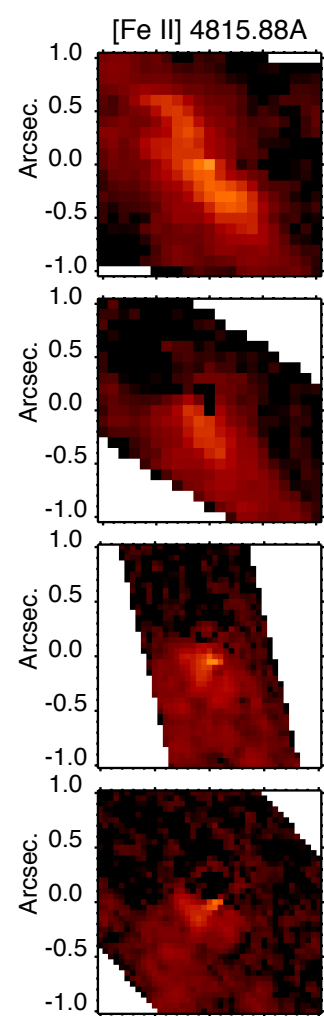

1.0
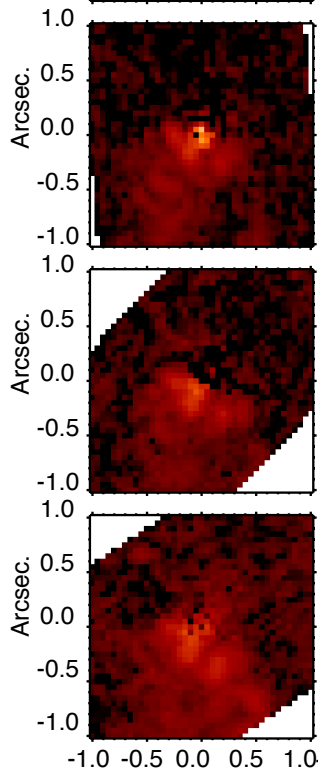

Arcsec.
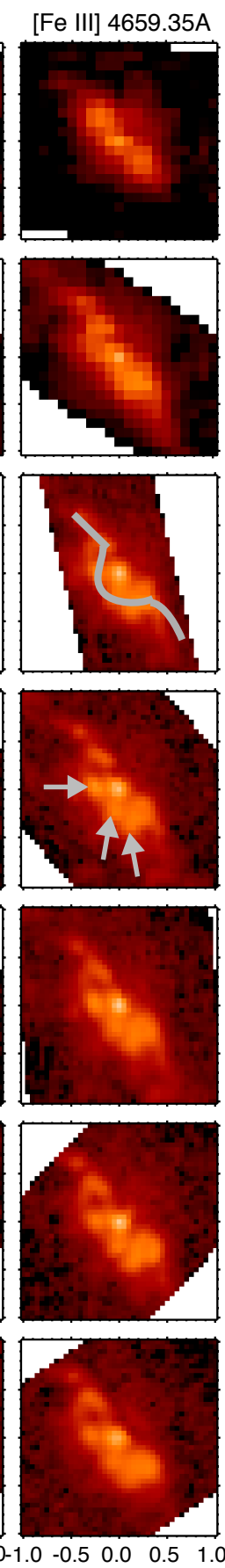

Arcsec.

4 e-16
Logarithmic Scale

Low State

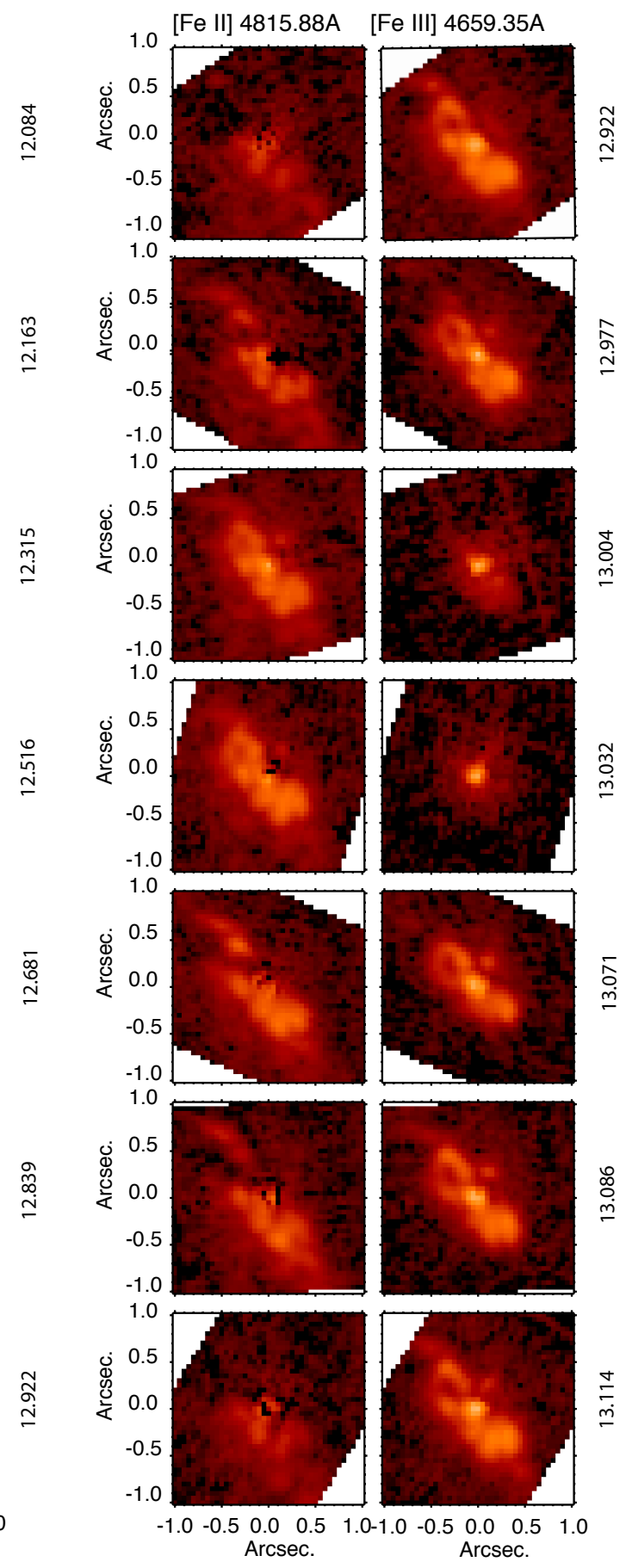

$-260 \mathrm{~km} / \mathrm{s}$

Figure 5. Changes in spatial structure from 2009.4 to $2014.2:-260 \mathrm{~km} \mathrm{~s}^{-1}$. Left: Iso-velocity slices of [Fe II] and [Fe III] for the seven mappings across the high-ionization state from $\phi=12.084$ to 12.922 . Right: Iso-velocity slices across the low-ionization state from $\phi=12.922$ to 13.114 (see Section 3.3.2 for description). Three arcuate structures, or claws, develop by $\phi=12.315$ (grey arrows, [Fe III] at $\phi=12.516)$ and persist across the high ionization state. Alternatively, a series of clumps define an $\Omega$-like shape in [Fe III] by $\phi=12.315$ that persists until $\phi=12.681$ (grey $\Omega$ in [Fe III], $\phi=12.315$ ) Then it develops into a figure-eight shape that disappears before periastron, but returns after the low-ionization state $(\phi=13.071$ to 13.114$)$. 
(iii) The $-260 \mathrm{~km} \mathrm{~s}^{-1}$ [Fe III] iso-velocity images in Fig. 5 reveal three claws: $0^{\prime \prime} .2$ at $80^{\circ}$ and $180^{\circ}$ plus $0^{\prime \prime} .4$ at $200^{\circ}$. By $\phi=12.977$, these claws show up in [Fe II], persist in [Fe II] through $\phi=13.086$, then begin to fade by $\phi=13.114$.

By contrast the [Fe III] structure, seen as a Crab-like structure from $\phi=12.315$ to 12.922 in $-140 \mathrm{~km} \mathrm{~s}^{-1}$ isovelocity images (Fig. 4) distorts into an $\Omega$-shape, rotated by $135^{\circ}$ (Fig. 5). With advancing phase, the [Fe III] shifts in form to a near-figure-eight at $\phi=12.977$, shrinks to emission at the position of $\eta$ Car plus faint, diffuse emission to the south and west, then re-emerges as the figure-eight by $\phi=13.071$. The [Fe III] again appears as a distorted $\Omega$ by $\phi=13.114$.

In both $\phi=12.084$ and 13.086 at $-260 \mathrm{~km} \mathrm{~s}^{-1}$ (Fig. 5), a remarkable arc pops up in [Fe II] pointing to the northeast, but with an apparent shadow cutting across from east to west about 0 .' 1 north of $\eta$ Car. At $\phi=13.086$, this [Fe II] arc appears to be shadowed before $\mathrm{PA}=45$ and after $\mathrm{PA}=$ $22^{\circ}$. The apparent shadows may indicate regions of higher photoionization adjacent the [Fe II] arc.

\subsubsection{The red-shifted structures}

The real surprise was revealed in maps across the lowionization state from $\phi=12.977$ to 13.032 (Figs. 6 and $7)^{6}$. Short, arcuate structures appear in [Fe II] extending from the central region as far out as 0 .' 9 ! As the FUV radiation disappeared from regions where iron was doubly-ionized across the high-ionization state, additional, unanticipated [Fe II] structures suddenly appeared (in Figs. 6 and 7, see the [Fe II] frames across $\phi=13.004$ to 13.086 compared to the [Fe II] frames across $\phi=12.315$ to 12.922). These [Fe II] structures are more extensive, i.e. are found in more distant regions, since the critical density is significantly lower than for [Fe III]

(i) The innermost, [Fe II] red-shifted arcuate-structures, seen at $\phi=12.315$ to be $0^{\prime \prime} .2$ radially separated from $\eta$ Car at $\mathrm{PA}=80^{\circ}$ and $180^{\circ}$, expand at $470 \mathrm{~km} \mathrm{~s}^{-1}$ across the highionization state (Teodoro et al. 2013). These innermost arcs are readily apparent through $\phi=12.977$, but become very diffuse from $\phi=13.004$ to 13.114 , following the shapes of the earlier, innermost structures seen one cycle earlier $\phi=$ 12.084 and 12.163 .

(ii) Three sets of [Fe II] arcuate structures, $0 .{ }^{\prime \prime} 2,0$. . 45 and 0 .' 7 separation from $\eta$ Car at $\mathrm{PA}=80^{\circ}$ and $180^{\circ}$ were visible across the high-ionization state (Teodoro et al. 2013), but additional small arcuate structures are present in [Fe III] to the northwest of $\eta$ Car from $\phi=12.315$ to 12.922 . The innermost set of [Fe II] and [Fe III] arcuate structures at $\phi=$ 12.315 to 13.839 form a nearly complete ring in figs. 6 and 7 .

(iii) Many more [Fe II] arcuate structures appear at greater separations from $\eta$ Car across the low-ionization state $(\phi=13.004$ to 13.071$)$.

(iv) Some of the additional [Fe II] structures, allowing for radial expansion, track with structures in [Fe III] across the high-ionization state from $\phi=12.315$ to 12.922 .

6 These iso-velocity slices do not include contamination from the weak [Fe II] or Fe II lines that contribute significantly above +300 $\mathrm{km} \mathrm{s}^{-1}$. See Appendix A. (v) The two inner structures are nearly complete rings in [Fe II] during the low-ionization state $(\phi=13.004$ to 13.071$)$

(vi) Two elongated structures are present in the +100 $\mathrm{km} \mathrm{s}^{-1}$ frames at $\phi=13.071$ through 13.114 in Fig. 6. The north-pointing structure is visible throughout the cycle in [Fe II] and appears in [Fe III] through $\phi=12.681$, but disappears by $\phi=12.839$, returning by $\phi=13.071$. The northeast-pointing structure is visible in [Fe II] at $40^{\circ}$ from $\phi=12.084$ to 12.839 but fades late in the high state and may be returning by $\phi=13.114$.

(vii) A third arc to the west-northwest at $0{ }^{\prime \prime} 2$ can be traced from $\phi=12.084$ to 12.977 at $+100 \mathrm{~km} \mathrm{~s}^{-1}$ but disappears by $\phi=13.004$. It is also visible in the $+220 \mathrm{~km} \mathrm{~s}^{-1}$ frames from $\phi=12.315$ to 12.681 (Fig. 7).

(viii) The arcuate structures in [Fe II] shrink with increasing velocity and converge, projected onto $\eta$ Car's position, by $+400 \mathrm{~km} \mathrm{~s}^{-1}$ (See Fig. B2 for complete coverage of these structures across all phases and red-shifted velocities). Models of the southeast quadrant shells suggest nested shells that were nearly spherical in shape (Teodoro et al. 2013).

\subsection{Difference mapping to show the photo-ionization and expansion effects across the high-ionization state}

For most of the binary period, the winds of $\eta$ Car B push the winds of $\eta$ Car A aside forming a large cavity in the current wind-wind collision structures. The current wind interaction structures, not reseolvable by $H S T$ have now resolved by VLTI (Weigelt et al. submitted). The long-term accumulation from many cycles leads to an extensive cavity that projects northwest of the binary. The FUV radiation from $\eta$ Car B escapes and ionizes much of the gas within the cavity and the outer portions of the compressed, modulated wind of $\eta$ Car A. Evidence of this asymmetry is demonstrated by the several arcseconds offset of the H II region from $\eta$ Car as imaged at radio wavelengths by Duncan \& White (2003).

As each periastron approaches, $\eta$ Car B dips deeply into the primary wind that absorbs most of the FUV radiation. Most of the [Fe III] emission structures disappear. $\eta$ Car B penetrates so deeply that it carves a tunnel-like cavity within the primary wind, then pushes and photo-ionizes the primary wind until the extensive cavity is expanded even further. The tunnel continues to expand above and below the orbital plane creating a compressed shell that persists for multiple cycles (Madura 2010; Gull et al. 2011; Madura 2012; Madura et al. 2013; Clementel et al. 2014, 2015a,b).

Multiple clumps appear in [Fe II], located to the southeast at red-shifted velocities (Figs. 6 and 7). Where FUV radiation impinges upon these compressed shells at, or near critical densities of $n_{e}=10^{7} \mathrm{~cm}^{-3}$, [Fe III] $4659.35 \AA$ is emitted. Elsewhere in the expanding shells, mid-UV from the primary star, and reprocessed FUV radiation contributed from the secondary star, leads to singly ionized iron. At densities close to $n_{e}=10^{6} \mathrm{~cm}^{-3}$, [Fe II] $4815.88 \AA$ is emitted. We use these emissions to trace clumpy arcs of compressed primary wind and to determine how these winds evolve across multiple 5.54-year periods.

One means to display these changes in wind structure is by normalized differencing of maps by using: Change = $\left(M_{P}-M_{A}\right) /\left(M_{P}+M_{A}\right)$, where $\mathrm{M}_{P}$ is a 2 -D map near pe- 
High State
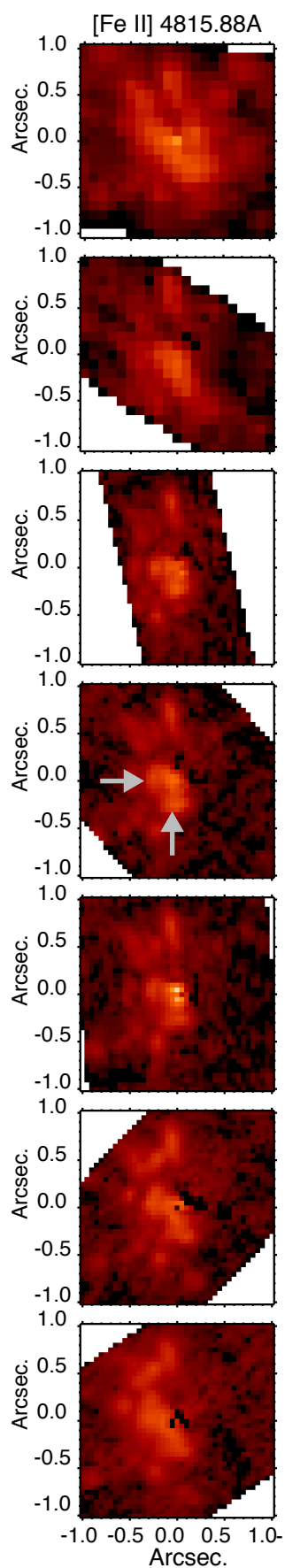
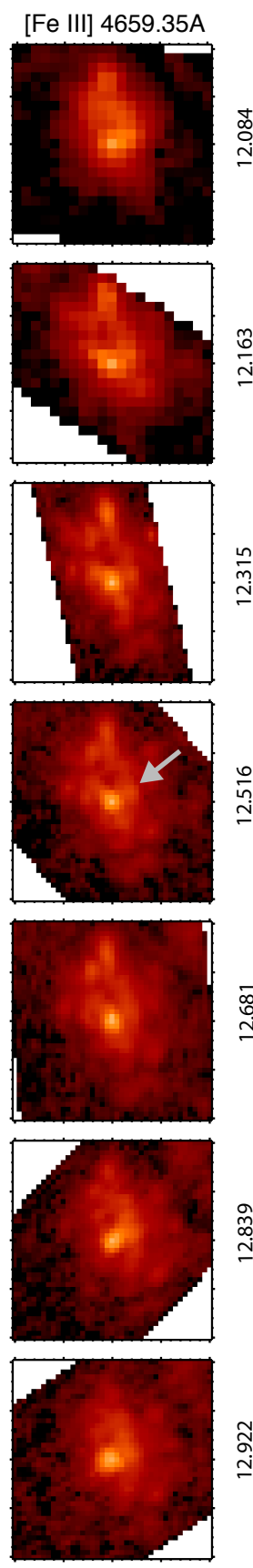

Logarithmic Scale

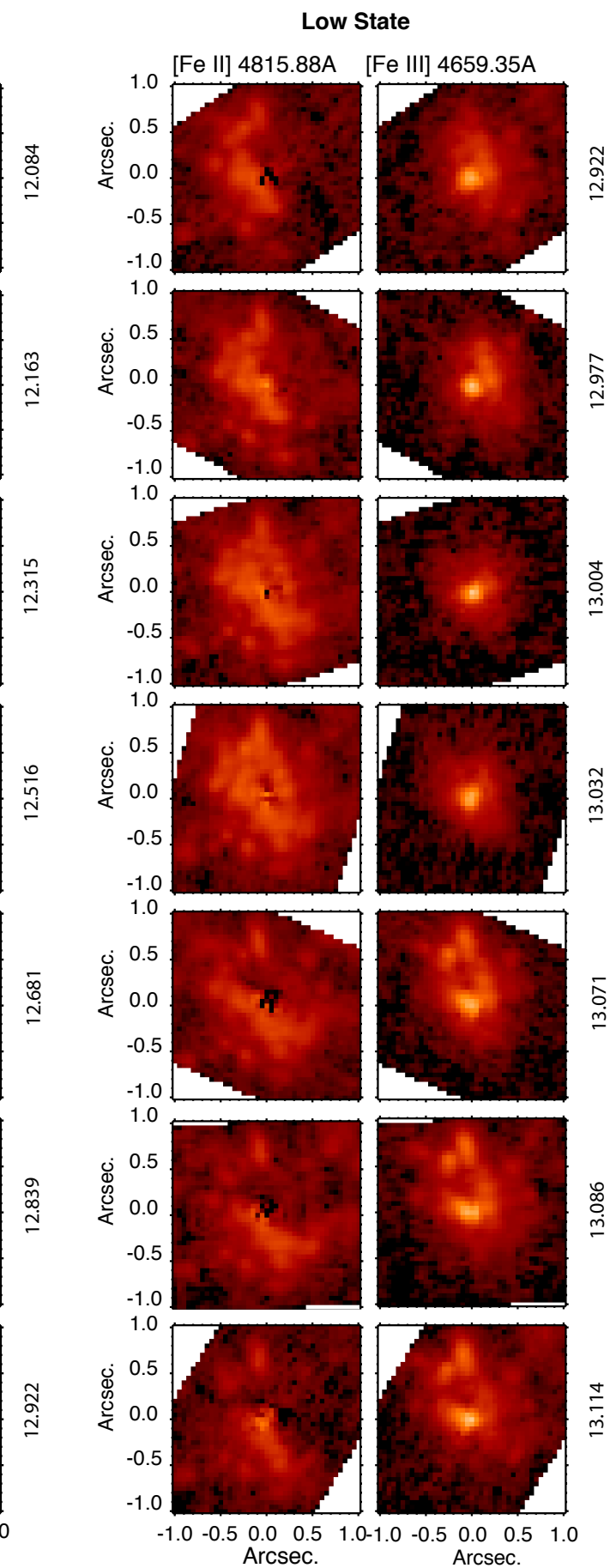

$+100 \mathrm{~km} / \mathrm{s}$

Figure 6. Changes in spatial structure from 2009.4 to $2014.2:+100 \mathrm{~km} \mathrm{~s}^{-1}$. Left: Velocity slices of [Fe II] and [Fe III] for the seven mappings across the high-ionization state from phases $\phi=12.084$ to 12.922 . Right: Seven mappings across the low-ionizaton state from phases $\phi=12.922$ to 13.114 . Red-shifted [Fe II] structures, situated on the far side of $\eta$ Car are visible, despite the intervening, foreground fossil winds. Two [Fe II] arcuate structures develop by $\phi=12.315$ (grey arrows, [Fe II] at $\phi=12.516$ ) and a [Fe III] arcuate structure at $\mathrm{PA}=280^{\circ}$ (grey arrow, [Fe III] at $\phi=12.516$ ) that form a nearly complete ring. Multiple clumps appear in [Fe II] during the low-ionization state $(\phi=13.004$ to 13.032). The Weigelt objects and hook structure, seen in low velocity (Fig. 3), are no longer present. 
High State
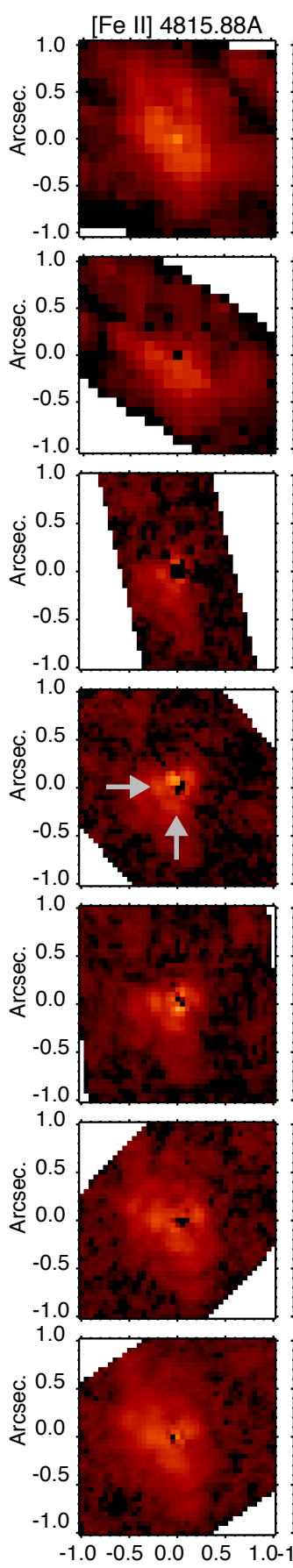

Arcsec.
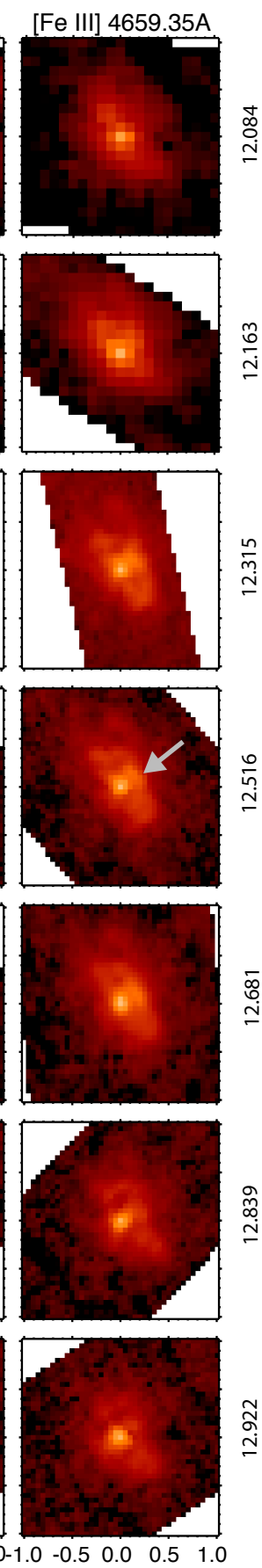

Arcsec.

4 e-16
Logarithmic Scale

Low State
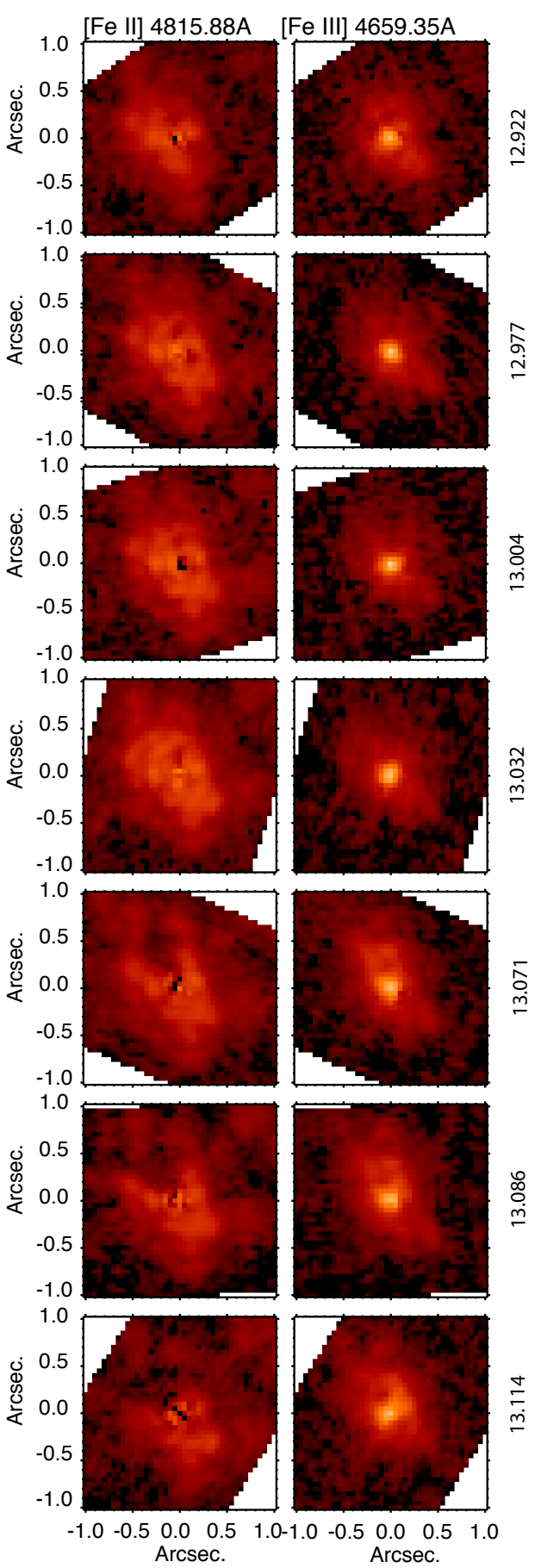

$+220 \mathrm{~km} / \mathrm{s}$

Figure 7. Changes in spatial structure from 2009.4 to $2014.2:+220 \mathrm{~km} \mathrm{~s}^{-1}$. Left: Velocity slices of [Fe II] and [Fe III] for the seven mappings across the high-ionization state from phases $\phi=12.084$ to 12.922 . Right: Seven mappings across the low-ionization state from phases $\phi=12.922$ to 13.114 . The [Fe II] emission is confined to the southeast quadrant across the last half of the high-ionization state ( $\phi=12.516$ to 12.922$)$. However across the low-ionization state, two large, continuous arcs form from north counter-clockwise to south, outlining large portions of the compressed primary wind. As seen in Fig. 6 , two [Fe II] arcuate structures develop by $\phi=12.315$ (grey arrows, [Fe II] at $\phi=12.516$ ) and a [Fe III] arcuate structure at PA $=280^{\circ}$ (grey arrow, [Fe III] at $\phi=12.516$ ) that form a nearly complete ring.A noticeable [Fe III] arc nearly completes the first shell ring formed in [Fe II], $0{ }^{\prime \prime} 2$ distance from $\eta$ Car, from $\phi=12.516$ to 12.922 . 
riastron, and $\mathrm{M}_{A}$ is a 2-D map near apastron. We provide iso-velocity slices of normalized differences in Figs. 8 and 9. Note that noise in the extreme velocity iso-velocity images displayed in Figs. 8 and 9 is significantly larger due to vey low signal.

\subsubsection{Difference maps in [Fe II]}

Related changes in structure occur in [Fe II] from apastron to periastron (Fig. 8, see also Teodoro et al. (2013)). The overall effect in the differencing images shows up as brightening (white) of $[\mathrm{Fe} \mathrm{II}]$ at periastron relative to apastron. In the radial direction from $\eta$ Car towards these red-shifted shells, the FUV is blocked by the primary wind in the current wind-wind interaction structures. Only the MUV can escape leading to singly-ionized iron.

Four large scale changes can be seen in the narrow velocity images:

(i) -100 to $+340 \mathrm{~km} \mathrm{~s}^{-1}$ : Structures of alternating bright/dark arcs appear counterclockwise from $\mathrm{PA}=90$ to $180^{\circ}$. These arcs are the remnants of compressed primarywind shells caused by the passage of $\eta$ Car B through the massive wind of $\eta$ Car A during periastron as analyzed by Teodoro et al. (2013).

(ii) -380 to $-100 \mathrm{~km} \mathrm{~s}^{-1}$ : Diffuse structures extend counterclockwise from $\mathrm{PA}=45$ to $90^{\circ}$ and 180 to $220^{\circ}$, slightly offset to the southeast from $\eta$ Car. These correlate with [Fe III]-bright structures at apastron and represent the outer, chaotic regions of the extensive wind-wind cavity.

(iii) +100 to $+340 \mathrm{~km} \mathrm{~s}^{-1}$ : Diffuse structures extend clockwise to the northwest, with an arcuate boundary grazing the position of $\eta$ Car. These structures also correlate with extended [Fe III]-bright structures at apastron and again are associable with outer, chaotic regions.

(iv) -100 to $+20 \mathrm{~km} \mathrm{~s}^{-1}$ : A lack of [Fe II] emission to the northwest that defines a large arcuate cavity with central edge grazing the position of $\eta$ Car. This darkened region follows the shape of the slow-moving structures as illustrated in Fig. 3. Additionally the shadows due to the 'ears' or filaments to the north at $\mathrm{PA}=35^{\circ}$ are described subsection 3.3.1.

\subsubsection{Difference maps in [Fe III]}

Changes in [Fe III] across the long, high-ionization state are represented in Fig. 9, which is a normalized difference between phases $\phi=12.516$ (apastron) and 12.922 (the last mapping before periastron when extensive [Fe III] is present). Each iso-velocity image is a $40 \mathrm{~km} \mathrm{~s}^{-1}$ velocity slice for the intervals from -380 to $+380 \mathrm{~km} \mathrm{~s}^{-1}$.

Most noticeable are two effects:

(i) Two white arcs, one 0.5 at $\mathrm{PA}=45^{\circ}$ and the other $0 . .5$ that $\mathrm{PA}=220^{\circ}$, appear in the -340 through $+20 \mathrm{~km} \mathrm{~s}^{-1}$ iso-velocity images in Fig. 9. At $-180 \mathrm{~km} \mathrm{~s}^{-1}$ less-defined interior dark pair of arcs appears signifying expansion of the [Fe III] structures across the 2.7-year interval between apastron and pre-periastron. These paired arcs can be followed to $+200 \mathrm{~km} \mathrm{~s}^{-1}$. A fainter, bright pair develops at velocities red-ward of $-20 \mathrm{~km} \mathrm{~s}^{-1}$. These arcs are $\mathrm{Fe}^{++}$portions of the compressed primary wind that continue to expand outward and apparently persist two cycles (eleven years) after being formed!

(ii) Irregular structures $0^{\prime \prime} .6$ centered on PA $=10^{\circ}$ first appear as two, thin, dark streaks by $-100 \mathrm{~km} \mathrm{~s}^{-1}$, become diffuse with red-shifting velocity until disappearance by +220 $\mathrm{km} \mathrm{s}^{-1}$. The darkening shifts clockwise to the east with increasing positive velocity and can be traced to $+180 \mathrm{kms}$. The apparent fading and shifting positions of [Fe III] emission are due to clumps of material deep within the complex structure that block the FUV radiation from $\eta$ Car B as it slowly moves across apastron. Hence the FUV radiation scans clockwise across distant structures.

\section{DISCUSSION}

The [Fe III] and [Fe II] structures paint a consistent picture of a series of partial fossil-wind shells with a large cavity, built up over multiple cycles, but which also contains obscuring, slow-moving, massive clumps of material originating from the two ejections during the 1840s and 1890s.

The [Fe III] maps reveal the highly-ionized $(>16.2 \mathrm{eV})$ portions of the fossil winds (Fig. B1). Across the highionization state, these structures extend counterclockwise from the northeast to the southwest with blue-shifts reaching to $-450 \mathrm{~km} \mathrm{~s}^{-1}$ (Figs.4, 5, and B1). Much fainter, somewhat red-shifted [Fe III] structures extend short distances to the northwest (Figs. B1, 3 and 6). All [Fe III] structures disappear across the low-ionization state except for faint emission at the position of $\eta$ Car (within the current cavity enclosed by the primary wind) and very faint, diffuse emission within 0 .' 3 to the northwest (small breakouts in the primary wind).

The [Fe II] maps reveal the low-ionized $(<7.9 \mathrm{eV})$ portions of the fossil winds (Fig. B2). Across the high-ionization state, relatively faint, blue-shifted [Fe II] structures, that are more diffuse than those seen in [Fe III], lay radially just beyond the [Fe III] structures (Figs. B2, 4 and 5). At redshifted velocities, multiple arcs appear in the [Fe II] (Figs. B2, 6 and 7). As analyzed by Teodoro et al. (2013), the arcs seen in the southeast across the multi-year high-ionization state were measured to expand as nearly spherical shells at $470 \mathrm{~km} \mathrm{~s}^{-1}$. The terminal wind velocity of $\eta$ Car A was determined to be $v_{\infty, A}=420 \mathrm{~km} \mathrm{~s}^{-1}$ (Groh et al. 2012). Modeling of the interacting winds by Madura et al. (2013) showed that the secondary wind interacting with the primary wind could accelerate these shells to $470 \mathrm{~km} \mathrm{~s}^{-1}$, consistent with the measured velocities of these fossil shells. The net effect would be variable expansion velocity of different portions of each shell dependent upon the total momentum imparted on the primary wind by the colliding secondary wind.

Across the high-ionization state, many [Fe II] arcs are visible in the southeast quadrant, but across the relatively brief low-ionization state, even more [Fe II] arcs appear surrounding $\eta$ Car (Figs. B2, 6 and 7). Three, possibly four, shells appear. Re-examination of the [Fe III] velocity maps confirm that some of the inner arcs are nearly complete, interior shells at low red-shifted velocities as a complementary portion appears in [Fe III] across the high-ionization state. Additional arcs and clumps, likely from shells even older than the relatively complete shells detected here, appear in [Fe II] across the low-ionization state at greater distances 
[Fe II] $\lambda 4815$

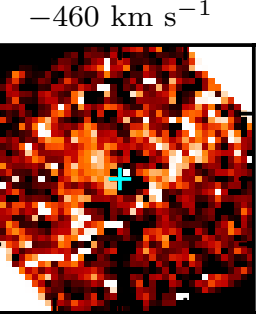

$-220 \mathrm{~km} \mathrm{~s}^{-1}$

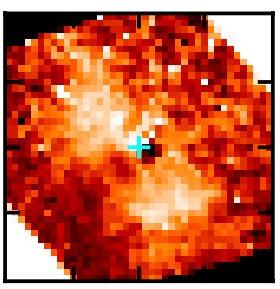

$+20 \mathrm{~km} \mathrm{~s}^{-1}$

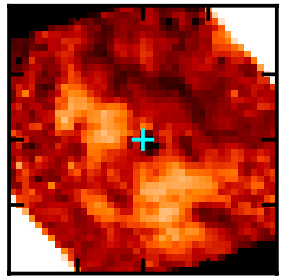

$+260 \mathrm{~km} \mathrm{~s}^{-1}$

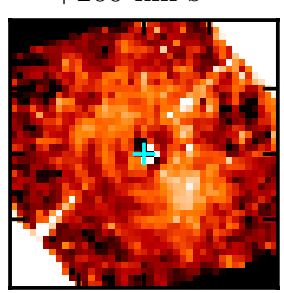

$-420 \mathrm{~km} \mathrm{~s}^{-1}$

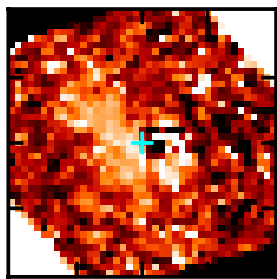

$-180 \mathrm{~km} \mathrm{~s}^{-1}$

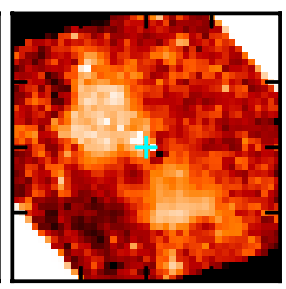

$+60 \mathrm{~km} \mathrm{~s}^{-1}$

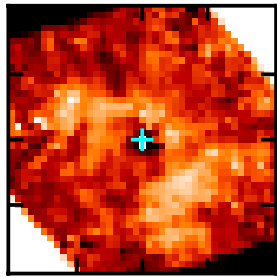

$+300 \mathrm{~km} \mathrm{~s}^{-1}$

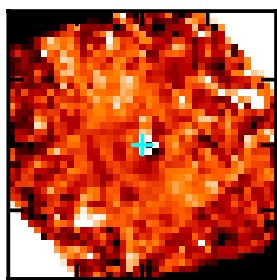

$-380 \mathrm{~km} \mathrm{~s}^{-1}$

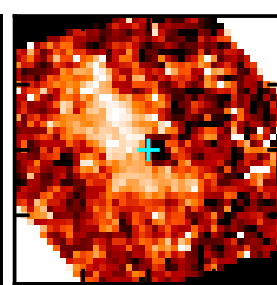

$-140 \mathrm{~km} \mathrm{~s}^{-1}$

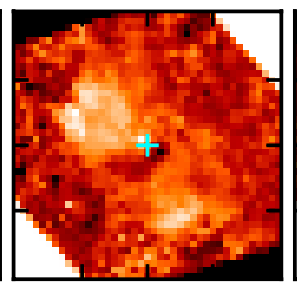

$+100 \mathrm{~km} \mathrm{~s}^{-1}$

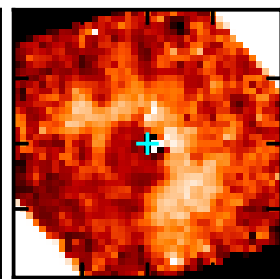

$+340 \mathrm{~km} \mathrm{~s}^{-1}$

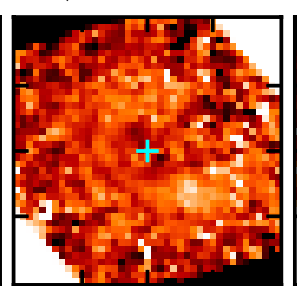

$-340 \mathrm{~km} \mathrm{~s}^{-1}$

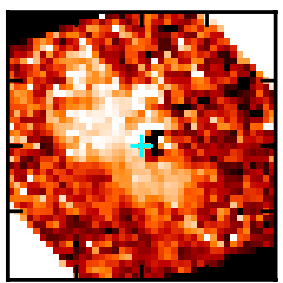

$-100 \mathrm{~km} \mathrm{~s}^{-1}$

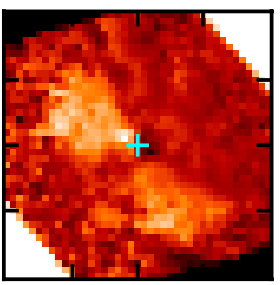

$+140 \mathrm{~km} \mathrm{~s}^{-1}$

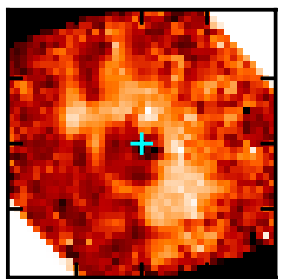

$+380 \mathrm{~km} \mathrm{~s}^{-1}$

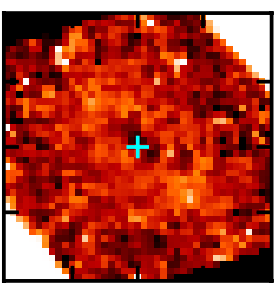

$-300 \mathrm{~km} \mathrm{~s}^{-1}$

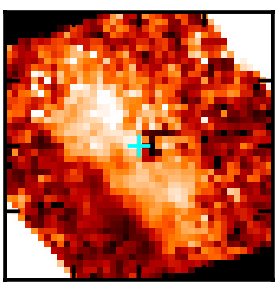

$-60 \mathrm{~km} \mathrm{~s}^{-1}$

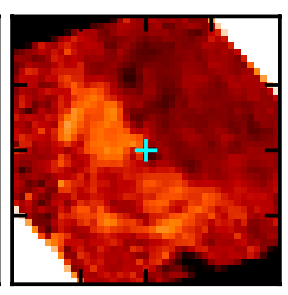

$+180 \mathrm{~km} \mathrm{~s}^{-1}$

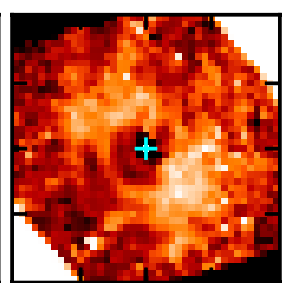

$+420 \mathrm{~km} \mathrm{~s}^{-1}$

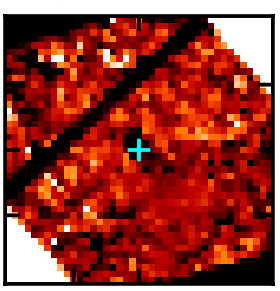

$-260 \mathrm{~km} \mathrm{~s}^{-1}$

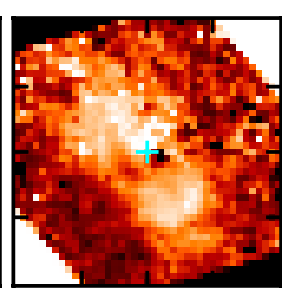

$-20 \mathrm{~km} \mathrm{~s}^{-1}$

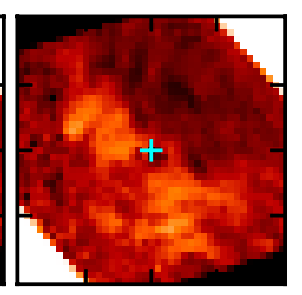

$+220 \mathrm{~km} \mathrm{~s}^{-1}$

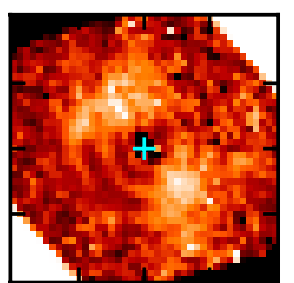

$+460 \mathrm{~km} \mathrm{~s}^{-1}$

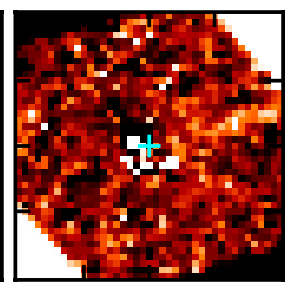

$-0.5$

0.0

Change

0.5

1.0

Figure 8. Expansion of the [Fe II] fossil shells between $\phi=12.516$ to 13.004 (2.7 years across the high-ionization state) and the light house effect. Displayed is the normalized difference between the fluxes of [Fe III] recorded at $\phi=12.516$, well into the high-ionization state, and at $\phi=13.004$, a half cycle later, just as the low-ionization state begins. Each frame is a $40 \mathrm{~km} \mathrm{~s}^{-1}$-wide slice of the [Fe III] profile starting at $-380 \mathrm{~km} \mathrm{~s}^{-1}$ in the upper left corner and ending at $+380 \mathrm{~km} \mathrm{~s}^{-1}$ in the lower right corner. White indicates regions where [Fe II] has increased.

from $\eta$ Car, likely due to relatively lower critical density for the transition.

The presence of these multiple [Fe II] shells, demonstrated by the multiple pairs of arcuate structures (Figs. 6 and 7) plus the nearly complete arcs in the normalized differences of (Fig. 8) strongly reinforce that the momentum balance of the winds has not changed substantially over the past several 5.5-year cycles. The periodic spacing of the arcuate structures, as measured by Teodoro et al. (2013), indicate near-uniform spacing. We suggest a limit of twenty percent to changes in the momentum balance. Additional observations should be made for confirmation.

The [Fe III] and [Fe II] mappings describe structures that are highly ionized on the near side and partially ion- ized on the far side of $\eta$ Car. Moreover the blue-shifted structures seen in [Fe III] are much more distorted and chaotic than the red-shifted structures seen in [Fe II]. Such can only be if the FUV source spends most of the orbit on the near side, and the same source provides the less-massive, faster wind. Hence, $\eta$ Car B, across the long, high-ionizaton state, centered upon apstron, the FUV and much faster, less massive wind source must be on the near side of $\eta$ Car A and the rapid passage through the massive wind of $\eta$ Car A by $\eta$ Car B must occur on the far side. This is contrary to the orbit proposed by Abraham et al. (2005) and Kashi \& Soker (2015), but consistent with models suggested by Pittard \& Corcoran (2002), Nielsen et al. (2007) and Madura (2010).

The iso-velocity images centered at +420 to +460 and 
[Fe III] $\lambda 4659$

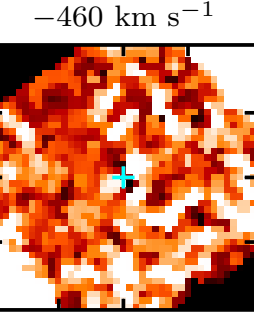

$-220 \mathrm{~km} \mathrm{~s}^{-1}$

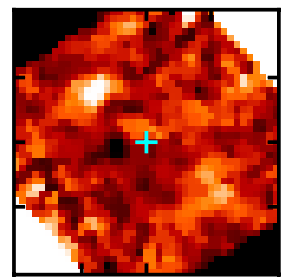

$+20 \mathrm{~km} \mathrm{~s}^{-1}$

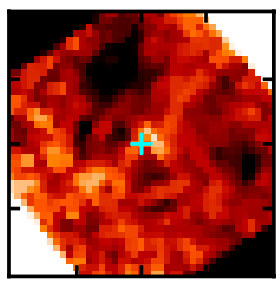

$+260 \mathrm{~km} \mathrm{~s}^{-1}$

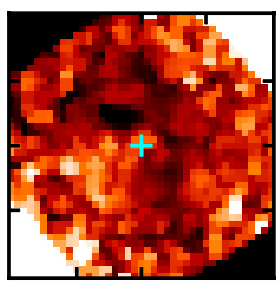

$-420 \mathrm{~km} \mathrm{~s}^{-1}$

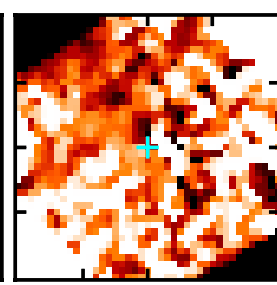

$-180 \mathrm{~km} \mathrm{~s}^{-1}$

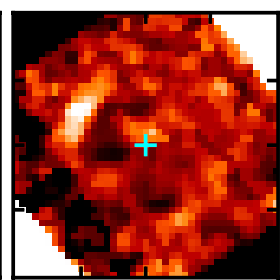

$+60 \mathrm{~km} \mathrm{~s}^{-1}$

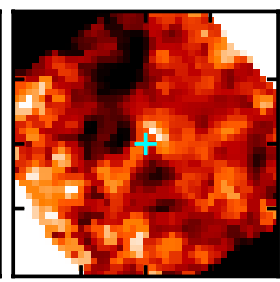

$+300 \mathrm{~km} \mathrm{~s}^{-1}$

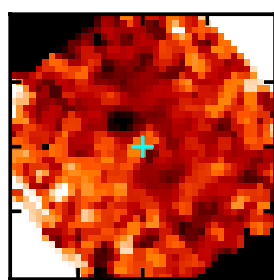

$-380 \mathrm{~km} \mathrm{~s}^{-1}$

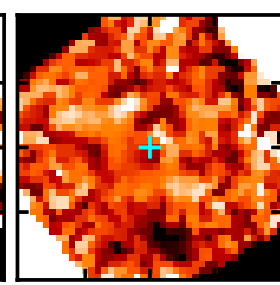

$-140 \mathrm{~km} \mathrm{~s}^{-1}$

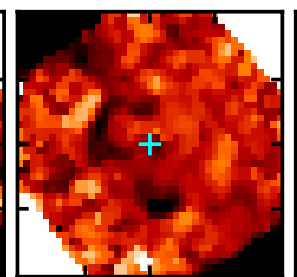

$+100 \mathrm{~km} \mathrm{~s}^{-1}$

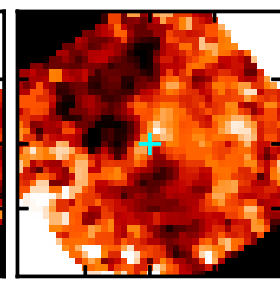

$+340 \mathrm{~km} \mathrm{~s}^{-1}$

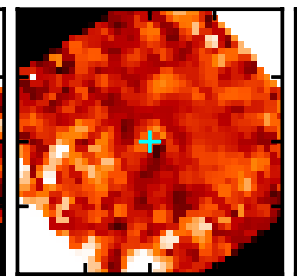

$-340 \mathrm{~km} \mathrm{~s}^{-1}$

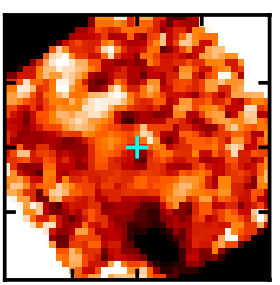

$-100 \mathrm{~km} \mathrm{~s}^{-1}$

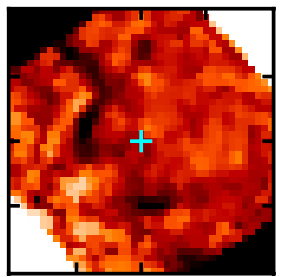

$+140 \mathrm{~km} \mathrm{~s}^{-1}$

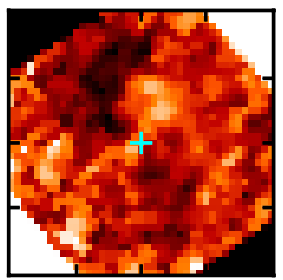

$+380 \mathrm{~km} \mathrm{~s}^{-1}$

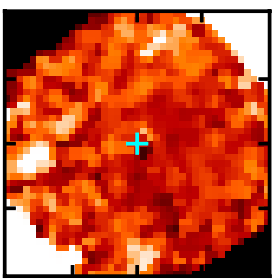

$-300 \mathrm{~km} \mathrm{~s}^{-1}$

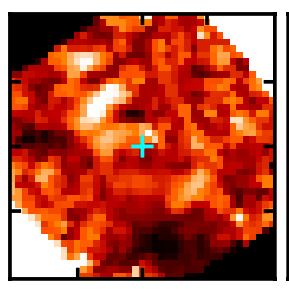

$-60 \mathrm{~km} \mathrm{~s}^{-1}$

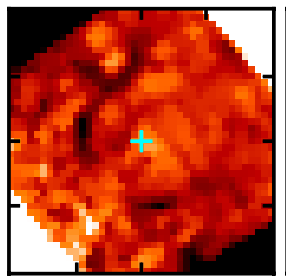

$+180 \mathrm{~km} \mathrm{~s}^{-1}$

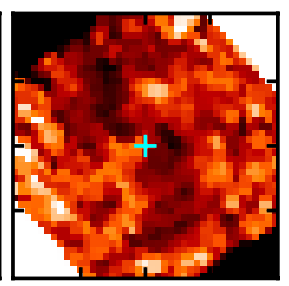

$+420 \mathrm{~km} \mathrm{~s}^{-1}$

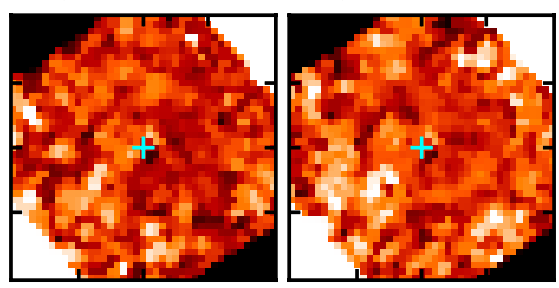

$-0.50$

$-0.25$

0.00

0.25

0.50

Figure 9. Shell expansion and the lighthouse effect of [Fe III $]$ emission between $\phi=12.516$ to 12.922. Each frame is a $40 \mathrm{~km} \mathrm{~s}^{-1}$-wide slice of the normalized difference starting at $-380 \mathrm{~km} \mathrm{~s}^{-1}$ in the upper left corner and ending at $+380 \mathrm{~km} \mathrm{~s}^{-1}$ in the lower right corner. [Fe III] intensity has dropped in regions displayed as black, and has increased in regions showing white. Expansion is revealed by paired white and black arcs to the northeast and southwest. The lighthouse effect, due to obstructing clouds deep within the structures that block the FUV, is especially prominent in the -60 to $+60 \mathrm{~km} \mathrm{~s}^{-1}$ frames. Structures about 0 .' 6 north by northwest darken while structures $0^{\prime \prime} 3$ due north relative to $\eta$ Car brighten in the -100 to $+100 \mathrm{~km} \mathrm{~s}^{-1}$ velocity range.

-420 to $-460 \mathrm{~km} \mathrm{~s}^{-1}$ show little emission (Figs. B1 and B2), since these intervals include the terminal velocity of the primary wind, $v_{\infty A}=420 \mathrm{~km} \mathrm{~s}^{-1}$ (Groh et al. 2012) and the partially-accelerated shell velocity, $470 \mathrm{~km} \mathrm{~s}^{-1}$ (Teodoro et al. 2013). The [Fe III] iso-velocity images for $-420 \mathrm{~km} \mathrm{~s}^{-1}$ show very weak diffuse emission extending to the northeast, bounded by $\mathrm{PA}=30$ to $70^{\circ}$ early and late in the highionization state (Fig. B1). The [Fe II] iso-intensity images show the same structure between $\phi=13.004$ and 13.032 (Fig. B2). A noticeable difference is in the [Fe II] frames for $\phi=12.084$ and 12.163 as there appears to be some weak [Fe II] emission not seen in the high velocity frames for $\phi=$ 13.086 and 13.114 .

Recently, Abraham et al. (2014) observed $\eta$ Car at sev- eral hydrogen transitions, $\mathrm{H}$ n $\alpha$, with the Atacama Large Millimeter/submillimeter Array $(A L M A)$. They postulated that the structures seen in selected H I $21 \alpha$ to $42 \alpha$ lines with $\approx 1^{\prime \prime}$ angular resolution were due to the formation of a baby Homunculus in the 1940's timeframe. These structures are quite similar in nature to 3 - $\mathrm{cm}$, radio-continuum structures imaged by Duncan \& White (2003) with the Australian Telescope Compact Array (ATCA). Both the continuum and the recombination-line structures appear to be offset to the northwest of the [Fe II] structures identified in the present paper.

We posit that these structures are the ionizedcomplement in the large cavity blown out over many cycles by the secondary wind and photo-ionized by the FUV 
radiation from the secondary star. As noted by Duncan \& White (2003), the radio continuum, while centered on $\eta$ Car, extends to the northwest growing and shrinking with binary phase over the 5.5 -year period. The [Fe III] structures mapped across the most recent cycle grow and shrink towards the northwest, but are confined to closer regions than the radio continuum. Likely this is because the [Fe III] emission reveals the relatively high-density regions $\left(\mathrm{n}_{e}=\right.$ $10^{7} \mathrm{~cm}^{-3}$ ) while the radio continuum originates from much lower, but larger regions in the extended wind cavity.

The recent observations of $\eta$ Car with the VLTI (Weigelt et al. submitted) of the $\mathrm{H} \mathrm{I} \mathrm{Br} \gamma$ line (angular resolution 6 mas, $\mathrm{R}=\lambda / \delta \lambda=12,000$ ) directly image the current wind-wind interactions. The $V L T I$ iso-velocity imagery supports the fossil wind structures discussed herein.

The Weigelt objects and related, slowly-moving structures, as suggested by their radial velocity (Fig. 3), are noticeable only on the near side. The regularly spaced arcs on the far side would not exist were there clumps moving at low velocities $\left(+10\right.$ to $\left.+100 \mathrm{~km} \mathrm{~s}^{-1}\right)$. The existence of the regularly-spaced, slow-moving, red-shifted arcs suggests that no analogs to the Weigelt blobs and extended structures exist on the far side. Does this suggest that the 1890s ejection occurred during periastron passage, leading to rapidly moving ejecta on the far side of $\eta$ Car, but that only some, very slow-moving ejecta flew out in our direction on the opposite side of the encounter? Is this another clue about the mechanism?

Curiously the detected clumps, of which the Weigelt objects are the brightest, are on the blue-shifted, highlyionized side of the system. Based upon what is seen in [Fe II], the red-shifted side of $\eta$ Car appears to be a fossil wind that is flowing uniformly over the past several 5.54-year periods with shell-like structures carved out by the rapid passage of $\eta$ Car B during each periastron event. No red-shifted, slowly moving clumps appear to be present.

\section{CONCLUSIONS}

A series of observations has been accomplished across one 5.54-year binary period. Using STIS on HST, we have systematically mapped the central region surrounding $\eta$ Car with 0 .' 13 angular resolution and $\mathrm{R}=\lambda / \delta \lambda=8,000$. Focusing on spatial- and velocity-differentiated structures seen in the light of [Fe III] $4659.35 \AA$ and [Fe II] $4815.88 \AA$, we have followed the changes in photo-ionization and expansion of multiple structures surrounding $\eta$ Car. We find these structures to be fossil remnants of wind-wind interactions remaining from periastron passages 5.5, 11.1, 16.6 and possibly 22.2 years previously.

Photo-ionization changes of the fossil structures are brought on by modulation of the FUV radiation that originates from $\eta$ Car B. The companions spend most of the orbit at $30 \mathrm{AU}$ separation (=1.3 mas at a distance of 2300 pc). For over five years of each binary period, much of the winds are highly-ionized and glow in [Fe III]. However across periastron, when the stars approach as close as $1.5 \mathrm{AU}$, $\eta$ Car B plunges deeply into the very massive, opticallythick, primary wind. For a few months, the FUV radiation from $\eta$ Car $\mathrm{B}$ is blocked by the primary wind. The wind structures become less-ionized leading to [Fe II] emission originating from much of the extended wind structures.

While the secondary wind interacts with the primary wind throughout the entire binary orbit, the secondary star passage deeply through the primary wind produces a tightly wound tunnel across each periastron, resulting in an expanding, compressed shell. Given the thermal velocities of the primary wind being much smaller than its terminal velocity, the compressed shell persists and expands outward from the binary. Both stars have such massive winds that, for several 5.5-year cycles, the densities of these compressions exceed $\mathrm{n}_{e} \approx 10^{7} \mathrm{~cm}^{-3}$, leading to forbidden emissions from $\mathrm{Fe}^{++}$and $\mathrm{Fe}^{+}$originating in portions of the shells. Hence we are able to trace the persistent fossil shells.

The fossil shells persist only for a fraction of the region surrounding $\eta$ Car, namely the far, red-shifted side where the periastron event occurs (Figs. B2, 6 and 7). The hot, secondary star spends most of the orbit on the near side of $\eta$ Car A, blowing an ionized, low-density cavity across each high-ionization state. Both the 3 -D hydrodynamical models of Okazaki et al. (2008); Parkin et al. (2009, 2011); Madura (2012); Madura et al. (2013); Clementel et al. (2014, 2015a) and the present observations support the concept that the cavity of the current wind-wind interaction eventually intersects with previous ionized cavities, building an ever larger structure that extends to the northwest of $\eta$ Car (Fig. 8). The blue-shifted [Fe III] structures extending from the northeast to the southwest (Figs. B1, 4 and 5) define the boundaries of this cavity in the form of highly-ionized primary wind structures.

The region between the well-defined [Fe II] shells in the red-shifted southwest and the blue-shifted [Fe III] structures include a more chaotic region. This is a transition region between the cavity blown by $\eta$ Car B and the modulated shells from the wind of $\eta$ Car A. In this region and within the wind-blown, large cavity, exist slow-moving clumps of material. The brightest of these are the Weigelt objects, C and D. Their proper motion and position suggests that they were ejected by the system in the 1890s event (Weigelt \& Kraus 2012).

The persistence of the multiple shells in $[\mathrm{Fe} \mathrm{II}]$ indicates:

(i) There are currently no large, slowly moving clumps of gas, like the Weigelt objects, on the far side of $\eta$ Car. This may be an important clue to the major ejection event of the 1890 s and possibly the $1840 \mathrm{~s}$. Was the event such that the faster moving ejecta was in all directions except towards apastron? Could the explosive event have been at or near periastron leading to an asymmetrical ejection not only in the polar regions but in the orbital plane?

(ii) The mass loss momentum and energy balance of the binary system has not changed significantly over the past several cycles. Does this indicate that $\eta$ Car has settled down for the near term? Could this indicate that the events of the 1840s and the 1890s were not pre-supernova events, but rather were the result of a stellar merger, or some other mechanism?

We leave the conclusion to the modelers and to future observations. 


\section{ACKNOWLEDGEMENTS}

TIM was supported by the NASA Postdoctoral Fellowship Program. TRG, TIM and MT received support from STScI grants 12013, 12750, 12508 and 13054 through June 2015. TRG also thanks Gerd Weigelt and the Max Planck Institute for Radioastronomy for delightful stays in the fall of 2015 and spring of 2016. AD acknowledges the continuing financial support from FAPESP. AFJM is grateful for financial support from NSERC (Canada) and FQRNT (Quebec). NDR acknowledges postdoctoral support by the University of Toledo and by the Helen Luedtke Brooks endowed Professorship. We gratefully thank Ms. Beth Perriello (STScI) for the extraordinary support scheduling visits at critical intervals that led to the success of this very challenging series of observing programs. We thank anonymous referee for helpful editorial comments. And most importantly, we thank the Eta Car Bunch, a truly innovative group extended across many locations but focused on one massive binary!

\section{REFERENCES}

Abraham Z., Falceta-Gonçalves D., Dominici T., Caproni A., Jatenco-Pereira V., 2005, MNRAS, 364, 922

Abraham Z., Falceta-Gonçalves D., Beaklini P. P. B., 2014, ApJ, 791,95

Chesneau O., et al., 2005, A\&A, 435, 1043

Clementel N., Madura T. I., Kruip C. J. H., Icke V., Gull T. R., 2014, MNRAS, 443, 2475

Clementel N., Madura T. I., Kruip C. J. H., Paardekooper J.-P., Gull T. R., 2015a, MNRAS, 447, 2445

Clementel N., Madura T. I., Kruip C. J. H., Paardekooper J.-P., 2015b, MNRAS, 450, 1388

Corcoran M. F., 2005, AJ, 129, 2018

Corcoran M., 2009, Monitoring Dynamical Mass Loss from Eta Car with the HETG, HST Proposal

Corcoran M., 2011, Monitoring Dynamical Mass Loss from Eta Car with the HETG and STIS: The Rise to Maximum, HST Proposal

Corcoran M. F., Ishibashi K., Swank J. H., Petre R., 2001, ApJ, 547,1034

Damineli A., 1996, ApJ, 460, L49

Damineli A., Conti P. S., Lopes D. F., 1997, New Astronomy, 2, 107

Damineli A., et al., 2008a, MNRAS, 384, 1649

Damineli A., et al., 2008b, MNRAS, 386, 2330

Davidson K., 1997, New Astronomy, 2, 387

Davidson K., Humphreys R. M., 1997, ARA\&A, 35, 1

Davidson K., Ebbets D., Weigelt G., Humphreys R. M., Hajian A. R., Walborn N. R., Rosa M., 1995a, AJ, 109, 1784

Davidson K., Ebbets D., Weigelt G., Humphreys R. M., Hajian A. R., Walborn N. R., Rosa M., 1995b, AJ, 109, 1784

Davidson K., et al., 1999, AJ, 118, 1777

Davidson K., Smith N., Gull T. R., Ishibashi K., Hillier D. J., 2001, AJ, 121, 1569

Davidson K., Mehner A., Humphreys R. M., Martin J. C., Ishibashi K., 2015, ApJ, 801, L15

Dorland B. N., Currie D. G., Hajian A. R., 2004, AJ, 127, 1052

Duncan R. A., White S. M., 2003, MNRAS, 338, 425

Falcke H., Davidson K., Hofmann K.-H., Weigelt G., 1996, A\&A, 306, L17

Fernández-Lajús E., et al., 2009, A\&A, 493, 1093

Groh J. H., Damineli A., 2004, Information Bulletin on Variable Stars, 5492

Groh J. H., Hillier D. J., Madura T. I., Weigelt G., 2012, MNRAS, 423, 1623
Gull T., 2011, Constraining the evolutionary state of the hot, massive companion star and the wind-wind collision region in Eta Carinae, HST Proposal

Gull T., 2012, Constraining the evolutionary state of the hot, massive companion star and the wind-wind collision region in Eta Carinae, HST Proposal

Gull T., 2013, Constraining the evolutionary state of the hot, massive companion star and the wind-wind collision region in Eta Carinae, HST Proposal

Gull T. R., Kober G. V., Nielsen K. E., 2006, ApJS, 163, 173

Gull T. R., et al., 2009, MNRAS, 396, 1308

Gull T. R., Madura T. I., Groh J. H., Corcoran M. F., 2011, ApJ, 743, L3

Heap S. R., et al., 1978, Nature, 275, 385

Hillier D. J., Allen D. A., 1992, A\&A, 262, 153

Hillier D. J., Davidson K., Ishibashi K., Gull T., 2001, ApJ, 553, 837

Hillier D. J., et al., 2006, ApJ, 642, 1098

Hofmann K., Weigelt G., 1988, A\&A, 203, L21

Iping R. C., Sonneborn G., Gull T. R., Massa D. L., Hillier D. J., 2005, ApJ, 633, L37

Kashi A., Soker N., 2015, preprint, (arXiv:1508.03576)

Kimble R. A., et al., 1998, ApJ, 492, L83

Madura T. I., 2010, PhD thesis, University of Delaware

Madura T. I. e. a., 2012, MNRAS, 420, 2064

Madura T. I., Groh J. H., 2012, ApJ, 746, L18

Madura T. I., et al., 2013, MNRAS, 436, 3820

Martin J. C., Davidson K., Koppelman M. D., 2006, astro$\mathrm{ph} / 0609295$

Mehner A., Davidson K., Ferland G. J., Humphreys R. M., 2010, ApJ, 710, 729

Mehner A., et al., 2015, A\&A, 578, A122

Nielsen K. E., Gull T. R., Vieira Kober G., 2005, ApJS, 157, 138

Nielsen K. E., Corcoran M. F., Gull T. R., Hillier D. J., Hamaguchi K., Ivarsson S., Lindler D. J., 2007, ApJ, 660, 669

Noll K., 2009, STIS ERO: Mapping the 3-D wind structure of Eta Carinae, HST Proposal

Okazaki A. T., Owocki S. P., Russell C. M. P., Corcoran M. F., 2008, MNRAS, 388, L39

Parkin E. R., Pittard J. M., Corcoran M. F., Hamaguchi K., Stevens I. R., 2009, MNRAS, 394, 1758

Parkin E. R., Pittard J. M., Corcoran M. F., Hamaguchi K., 2011, ApJ, 726, 105

Pittard J. M., Corcoran M. F., 2002, A\&A, 383, 636

Richardson N. D., Gies D. R., Gull T. R., Moffat A. F. J., St-Jean L., 2015, AJ, 150, 109

Richardson N. D., et al., 2016, preprint, (arXiv:1606.03655)

Smith N., 2004, MNRAS, 351, L15

Smith N., Morse J. A., Collins N. R., Gull T. R., 2004, ApJ, 610, L105

Steffen W., et al., 2014, MNRAS, 442, 3316

Steiner J. E., Damineli A., 2004, ApJ, 612, L133

Teodoro M., Madura T. I., Gull T. R., Corcoran M. F., Hamaguchi K., 2013, ApJ, 773, L16

Teodoro M., et al., 2016, ApJ, 819, 131

Walborn N. R., 2012, in Davidson K., Humphreys R. M., eds, Astrophysics and Space Science Library Vol. 384, Eta Carinae and the Supernova Impostors. p. 25, doi:10.1007/978-1-46142275-4_2

Weigelt G., Ebersberger J., 1986, A\&A, 163, L5

Weigelt G., Kraus S., 2012, in Davidson K., Humphreys R. M., eds, Astrophysics and Space Science Library Vol. 384, Eta Carinae and the Supernova Impostors. p. 129, doi:10.1007/978-1-4614-2275-4_6

Weigelt G., et al., 1995, in Niemela V., Morrell N., Feinstein A., eds, Revista Mexicana de Astronomia y Astrofisica Conference Series Vol. 2, Revista Mexicana de Astronomia y Astrofisica Conference Series. p. 11 
[Fe III] $\lambda 4659$ from -500 to $+500 \mathrm{~km} \mathrm{~s}^{-1}$

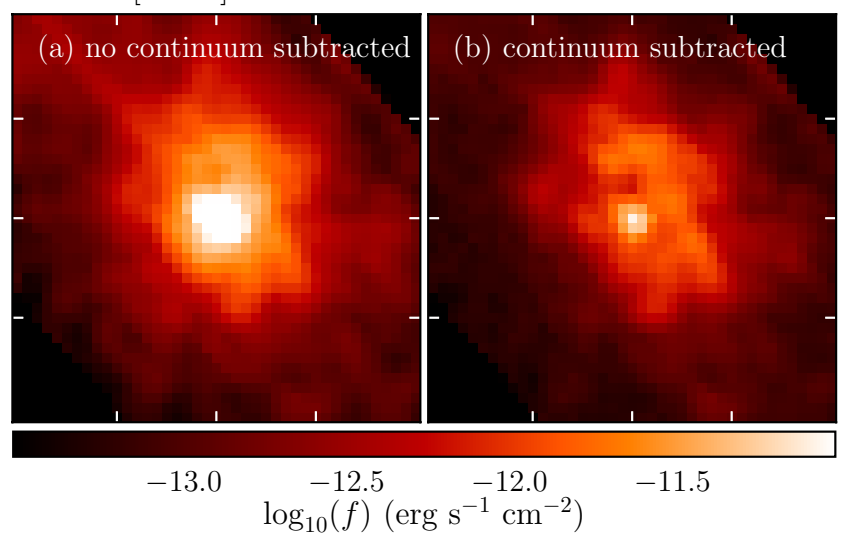

[Fe III] $\lambda 4659$ at $-140 \mathrm{~km} \mathrm{~s}^{-1}$

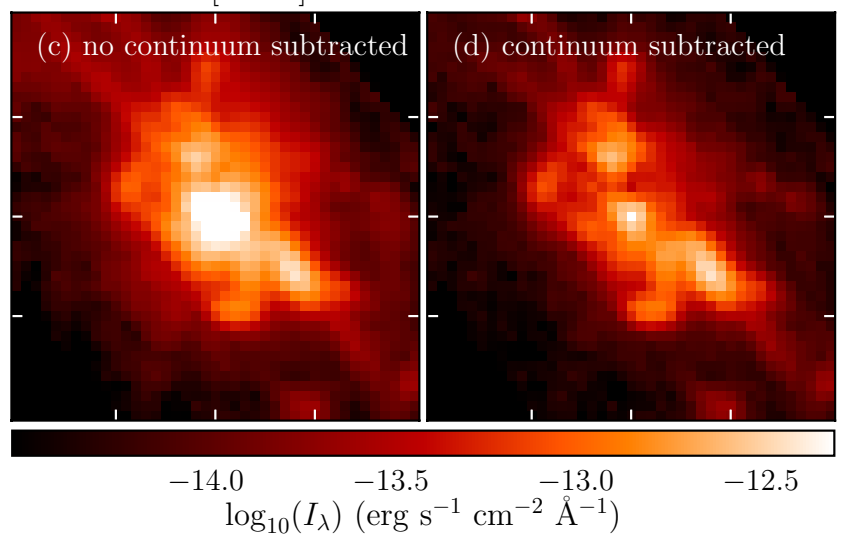

Figure A1. Two examples of continuum subtraction for [Fe III] 4659 $\AA$ recorded during the mapping visit near apastron, $\phi=12.516$. a: Image slice, $1000 \mathrm{~km} \mathrm{~s}^{-1}$ wide, which includes continuum. b: Same image slice with continuum subtracted. c: Image slice $40 \mathrm{~km} \mathrm{~s}^{-1}$ wide centered at $-140 \mathrm{~km} \mathrm{~s}^{-1}$ including continuum. d: Same image slice with continuum subtracted. The dynamic range, as demonstrated by the color bar at the bottom of each figure, is set to maximize the information content. The angular size of all of these images is $2^{\prime \prime} \times 2^{\prime \prime}$. The velocity interval, $40 \mathrm{~km} \mathrm{~s}^{-1}$, is comparable to the spectrographic resolving power, $R=\lambda / \delta \lambda=8000$ or $33 \mathrm{~km} \mathrm{~s}^{-1}$ in velocity.

Woodgate B. E., et al., 1998, PASP, 110, 1183

Zethson T., 2001, PhD thesis, Lunds Universitet (Sweden)

Zethson T., Johansson S., Hartman H., Gull T. R., 2012, A\&A, 540, A133

\section{APPENDIX A: DATA REDUCTION}

Continuum emission is the major contribution to spectra recorded when the STIS aperture is centered on, or near, $\eta$ Car and is also a very significant component due to scattered starlight in the surrounding nebular structures. The continuum flux must be subtracted in order to isolate emission from the extended wind-wind structures. Indeed, the spectral region from 4563 to $4749 \AA$, recorded with the G430M grating set at the central wavelength, $4706 \AA$, has very few spectral intervals free from contributions from broadened stellar lines and narrow nebular lines. While many procedures were tested to subtract the continuum, the more successful approach was a multivariate fit across the recorded spectral region. By comparison, Martin et al. (2006), Mehner et al. (2015) and Teodoro et al. (2016) referenced measurements to a short interval at 4741 to $4743 \AA$ and 4595 to $4605 \AA$. Test subtractions confirmed that the spectral interval around $4600 \AA$ is contaminated by spatially resolved emission and absorption structures, and hence is unsuitable for continuum reference.

Our objective was to obtain velocity information for selected spectral lines. We found the best approach was to construct data cubes, with coordinates of right ascension, declination and velocity, from the mapping spectra with appropriate subtraction of continuum. Examples of image slices before and after continuum subtraction are presented in Fig. A1. Each longslit spectrum was reduced by standard $S T I S$ data reduction tools. A routine, written using Interactive Data Language $\left(\mathbf{I D L}^{7}\right)$, provided continuum subtraction from each spectrum and resampled the multiple spectra into 3-D data cubes with velocity referenced to the transition wavelength: in this case, the [Fe III] $4659.35 \AA$ and the [Fe II] $4815.88 \AA$ lines.

Subtraction of the continuum and careful selection of the velocity interval brings out considerable detail on the fossil wind structures as demonstrated in Fig. A1. Images displayed in A1.a and .b extend from -500 to $+500 \mathrm{~km} \mathrm{~s}^{-1}$ centered on [Fe III] 4659.35 $\mathrm{A}$. In Fig. A1.a., the stellar core is surrounded by a diffuse haze and a hint of structure. Within each individual long-aperture, spatially resolved spectrum, a spectrum was extracted for each spatial element along the aperture. Then a slowly varying function was fitted to the spectrum to obtain the continuum level which was then subtracted. Considerably more spatial structure then appears as shown in Fig. A1.b but which still spans the -500 to +500 $\mathrm{km} \mathrm{s}^{-1}$ range. Narrowing the velocity range to $40 \mathrm{~km} \mathrm{~s}^{-1}$, slightly more than the $33 \mathrm{~km} \mathrm{~s}^{-1}$ resolution of the spectroscopic setup, reveals considerable differences in structures. In Fig. A1.c, which has no continuum subtracted, the stellar core dominates the image, but in Fig. A1.d, continuum has been subtracted which greatly suppresses the stellar core. Much more structural detail can be traced.

All images in Figs. 3 through 7, B1 and B2 have been continuum subtracted and normalized to the brightest structures. All images have a dynamic range from $\log _{10}(4 \times$ $10^{-16}$ to $\left.4 \times 10^{-13} \mathrm{erg} \mathrm{cm}^{-2} \mathrm{sec}^{-1}\right)$.

\section{APPENDIX B: THE GLOBAL CHANGES OF [FE III] AND [FE II] ACROSS ONE BINARY PERIOD}

The velocity range, intervals and central velocities were chosen based upon observed structures. As demonstrated by Gull et al. (2009, 2011), the emission line structures are visible throughout the velocity range between -470 to +470 $\mathrm{km} \mathrm{s}^{-1}$, consistent with the models discussed by Groh et al. (2012). We find no evidence of strong emission in the [Fe III] or [Fe II] lines blue-ward beyond the $-460 \mathrm{~km} \mathrm{~s}^{-1}$ band

7 IDL is a trademark of Exelis Visual Information Solutions, Inc,; http://www.exelisvis.com/ProductServices/IDL.aspx 
(range from -480 to $-440 \mathrm{~km} \mathrm{~s}^{-1}$ ). We chose to display the velocity images in $40 \mathrm{~km} \mathrm{~s}^{-1}$ intervals as that showed sufficient changes in structure in a finite number of frames. While Smith (2004) measured the velocity range of $\mathrm{H}_{2}$ in the Homunculus and estimated the system velocity, $V_{\text {sys }}=-8.1 \pm 1$ $\mathrm{km} \mathrm{s}^{-1}$, we note that Weigelt $\mathrm{D}$, as analyzed by Zethson (2001); Zethson et al. (2012) is centered at $-45 \mathrm{~km} \mathrm{~s}^{-1}$. Integrated profiles of [Fe III] and [Fe II] from the $2^{\prime \prime} \times$ $2^{\prime \prime}$ maps produce narrow line components centered at -40 $\mathrm{km} \mathrm{s}^{-1}$ with half-intensity levels at -80 and $+20 \mathrm{~km} \mathrm{~s}^{-1}$.

As we desired to track the slowly moving gas relative to the fossil wind structures, for the velocity range displays in Figs. B1 and B2, we displayed images centered at -60 and $-20 \mathrm{~km} \mathrm{~s}^{-1}$, on both sides of the slowly moving gas and extended the display range from -500 to $+500 \mathrm{~km} \mathrm{~s}^{-1}$.

Instrumental and telescope diffraction effects minimally affect the velocity frames displayed in Figs. B1 and B2. The measured full width at half maximum width of the core component of $\eta$ Car averaged 0.' 13 with small variations between visits. These critically-phased observations were recorded at arbitrary PAs, dictated by HST solar panel orientations. While a noticeable charge-transfer-inefficiency (CTI) trail, due to the aging CCD, and a diffraction ring are present in the reduced, broad-band integrated STIS imagery, continuum subtraction cancels out these contributions. Some hot column effects remain in the data, most noticeably in [Fe II] $+420 \mathrm{~km} \mathrm{~s}^{-1}$ iso-velocity frames (Fig. B2). Despite large changes in PA from visit to visit, the [Fe III] and [Fe II] structures persist, changing very slowly, corresponding to changes in expanding physical structures at their photoionization and critical densities. 


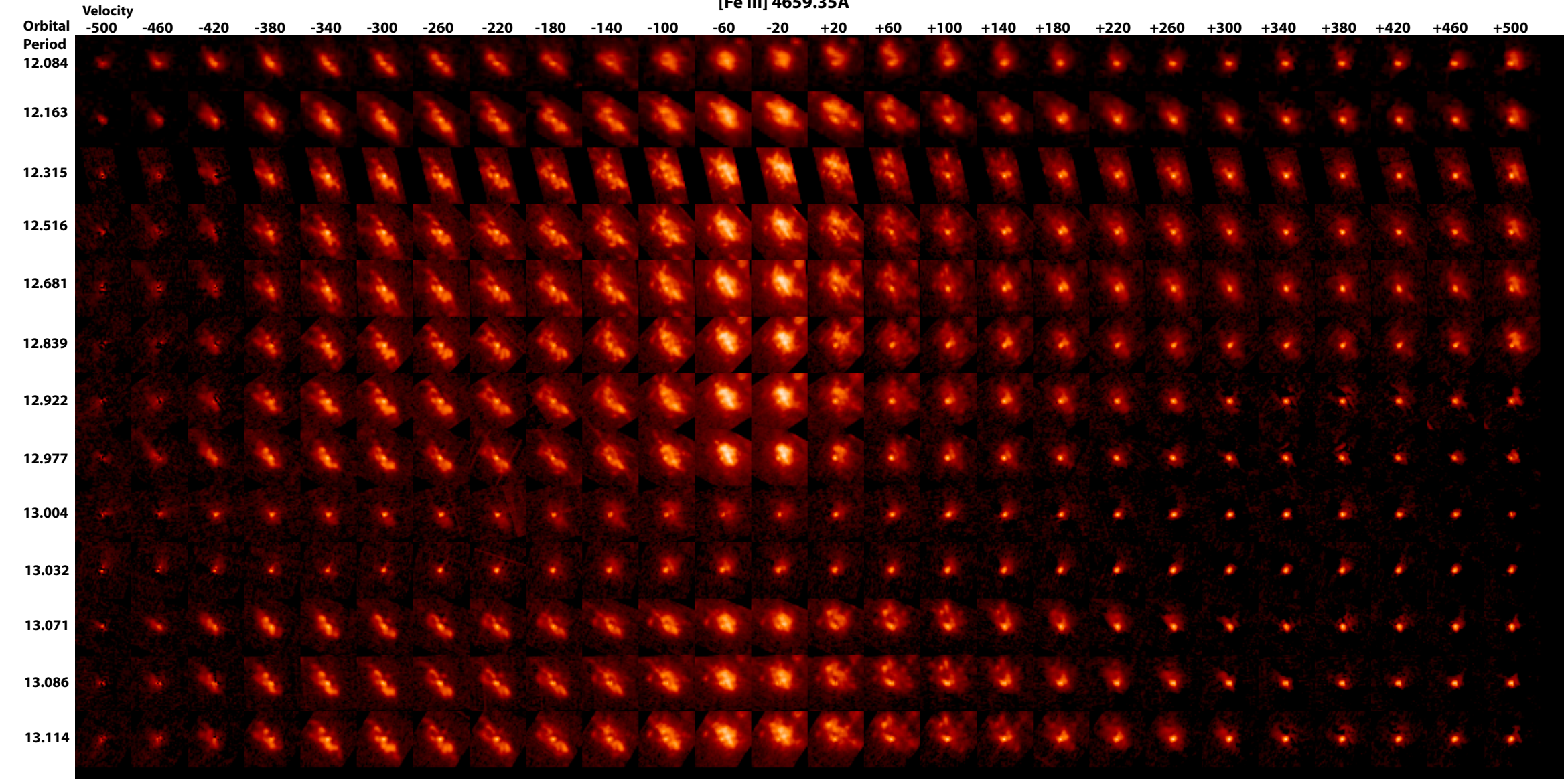

Figure B1. Evolution of highly ionized structures across the long high-ionization state through the low-ionization state and recovery: [Fe III] 4659.35 $\AA$ mappings from phases $\phi=\mathbf{1 2 . 0 8 4}$ to $\mathbf{1 3 . 1 1 4}$. The [Fe III] emission traces relatively dense $\left(\mathrm{n}_{e} \approx 10^{7} \mathrm{~cm}^{-3}\right)$, gaseous regions that are directly photo-ionized by $>16.2$ eV FUV radiation. The initial mapping (top row $\phi=12.084$ ) shows the system in recovery from the low-ionization state induced by the periastron passage of $\eta$ Car B, the UV source, deep within the extended wind of $\eta$ Car A. The high-ionization state is well developed by $\phi=12.163$ and stays bright through 12.839 , then rapidly fades to a stellar-like core by $\phi=13.004$, a few days after the onset of the low-ionization state. By $\phi=13.071$, recovery towards the high-ionization state is occurring. The extended complex at -60 and $-20 \mathrm{~km} \mathrm{~s}{ }^{-1}$, which is the diffuse structure associated with the evolved Weigelt objects, develops a hook-shaped structure to the north and west of the central object. Note the lack of bright clumps in the +20 and $+60 \mathrm{~km} \mathrm{~s}{ }^{-1}$ velocity columns. Two major effects occur across the 5.54-year period: 1) expansion of arcs radially outward from $\eta$ Car, and 2) clockwise-tangential shifts of ionized regions due to velocity columns. Two major effects occur across the 5.54-year period: 1) expansion of arcs radially outward from $\eta$ Car, and 2) clockwise-tangential shifts of ionized regions due to
movement of $\eta$ Car B within the cavity and intervening absorbing material. Detailed explanations of these many structures must await 3-D hydrodynamic modeling with radiative transfer. This is a display of the central $2^{\prime \prime} \times 2^{\prime \prime}$ region around $\eta$ Car. Each continuum-subtracted frame, centered on $\eta$ Car, is an iso-velocity slice 40 km $\mathrm{s}^{-1}$ wide, displayed with the range from $\log _{10}\left(4 \times 10^{-16}\right)$ to $\log _{10}\left(4 \times 10^{-13}\right) \mathrm{erg} \mathrm{sec}^{-1} \mathrm{~cm}^{-2}$. Each row displays velocity slices for a specific orbital phase recorded from June $2009(\phi=12.084)$ to March $2015(\phi=$ 13.114 ) and each column displays velocity slices ranging from -500 to $+500 \mathrm{~km} \mathrm{~s}^{-1}$ at increments of $40 \mathrm{~km} \mathrm{~s}^{-1}$. 


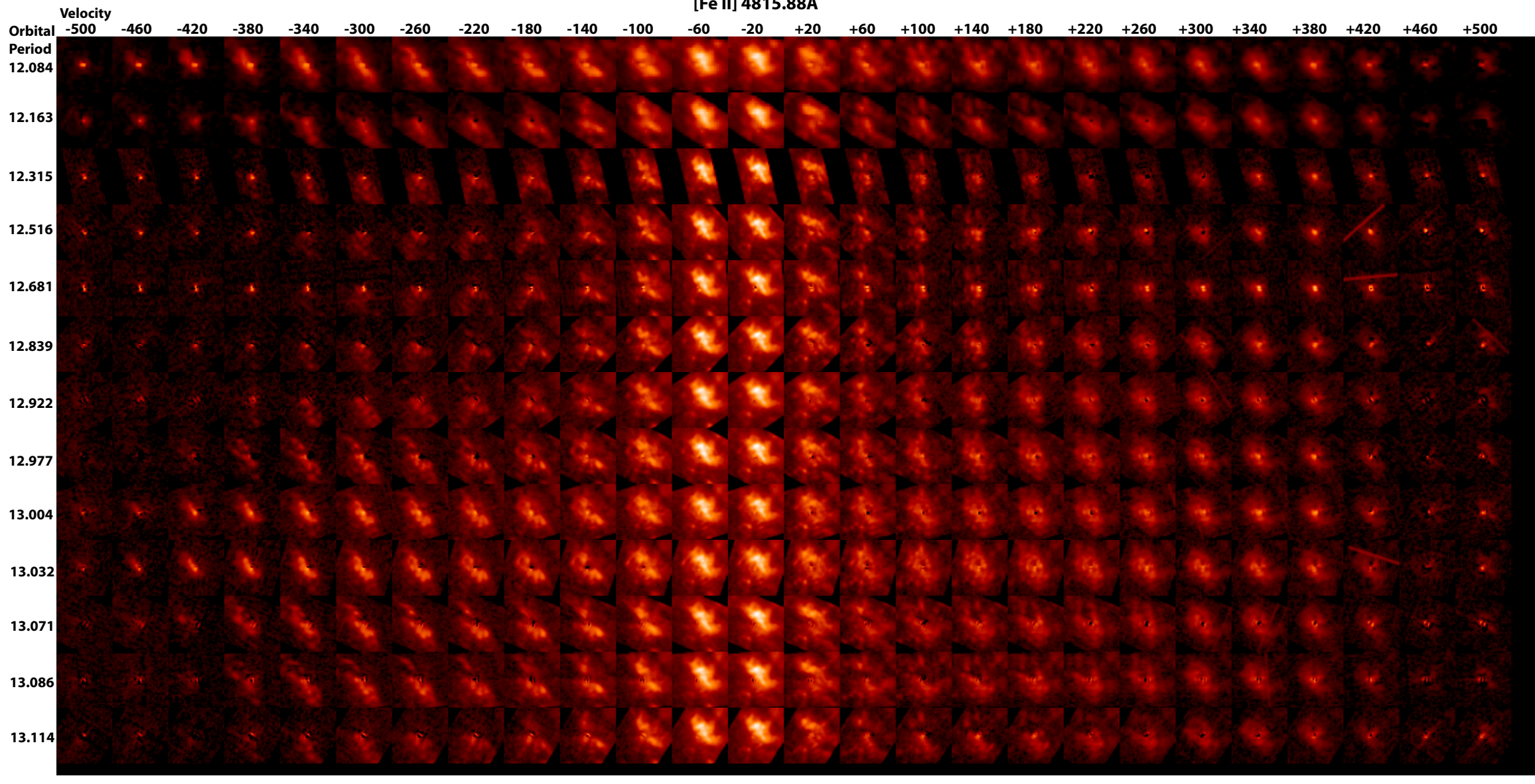

Figure B2. Evolution of low-ionized structures across the long high-ionization state through the low-ionization state and recovery: [Fe II] 4815.88 $\AA$ mappings from phase $\phi=\mathbf{1 2 . 0 8 4}$ to $\mathbf{1 3 . 1 1 4}$. The [Fe II] traces regions where $\mathrm{n}_{e}=2 \times 10^{6} \mathrm{~cm}^{-3}$, lower than traced by the [Fe III] emission, and where iron is photo-ionized to Fe ${ }^{+}$by FUV radiation with energies exceeding $7.9 \mathrm{eV}$. Throughout the 5.54-year period, the slowly moving gas $\left(-60\right.$ and $\left.-20 \mathrm{~km} \mathrm{~s}^{-1}\right)$ is well defined as a hook-shaped structure to the north and west of $\eta$ Car. At $\phi=12.084$ (first row) relatively bright structures are present from -460 to $-420 \mathrm{~km} \mathrm{~s}^{-1}$. By $\phi=12.163$, the high velocity components are fading and continue to fade (2.032, then begins to fade by $\phi=13.071$. Especially at red-shifted velocities, many clumps appear in [Fe II] extending out to the edges of the $2^{\prime \prime} \times 2^{\prime \prime}$ frame. Likely these are clumps that are photo-ionized to Fe ${ }^{++}$across the high-ionization state, but have densities, 
The rows of the two mosaics are paired by binary phase for comparison. The mappings began in June 2009 at $\phi=$ 12.084 , as the system was recovering from the 2009.1 periastron event and ended 5.7 years later in March 2015 at $\phi=13.114$, just over one cycle later. Two mappings are very close to one cycle separation: $\phi=12.084$ and 13.086 . A second near-pairing was accomplished with $\phi=12.163$ and 13.114. However both the $\phi=12.084$ and 12.163 mappings were accomplished with $0{ }^{\prime \prime} 1$ sampling, not the optimal sampling of 0. . 05 . Hence comparisons are limited due to nonoptimal sampling in the first two visits. Mappings were done at regular intervals across the long, high-ionization state, where spectrophotometry over past cycles showed relatively little change and, at critical phases, based upon predictions from the 3-D hydrodynamical models (Madura 2012; Madura et al. 2013).

The two lines selected for this study are relatively clear of weaker emission lines and broad stellar absorption features (Appendix A). The [Fe II] velocity range from -500 to $+500 \mathrm{~km} \mathrm{~s}^{-1}$ has no contamination from weak nebular emission lines, identified in spectra of Weigelt D (Zethson et al. 2012). However the [Fe III] velocity range has three weak lines that contribute flux at velocities beyond +340 $\mathrm{km} \mathrm{s}^{-1}$. While weaker relative to the [Fe III] line by an order of magnitude, an [Fe II] line at $+364 \mathrm{~km} \mathrm{~s}^{-1}$ and a (permitted) Fe line at $+412 \mathrm{~km} \mathrm{~s}^{-1}$ remain across the lowionization state when [Fe III] disappears. As described in Appendix B, the [Fe II] 4815.88§ emission line in the form of a data cube was shifted in velocity and adjusted in flux to null out contributions from these two lines in Fig. B1. Hence while the mosaics are suitable for comparison at all velocities, the reader must be aware that a qualitative correction has been made in the [Fe III] velocity frames at velocities red-shifted beyond $+340 \mathrm{~km} \mathrm{~s}^{-1}$.

Complex spatial structures in [Fe III] are visible across the long, high-ionization state (Fig. B1, $\phi=12.084$ to 12.977 and 13.071 to 13.114 ) that disappear during the relatively short, low-ionization state $(\phi=13.004$ to sometime beyond 13.032).

While $[\mathrm{Fe} \mathrm{II}]$ is bright across the entire orbital period in the velocity bands centered on -60 and $-20 \mathrm{~km} \mathrm{~s}^{-1}$ (Fig. B2), the blue-shifted and red-shifted velocity bands are brightest in $[\mathrm{Fe} \mathrm{II}]$ across the low-ionization state and fade considerably across the high-ionization state (a more detailed discussion on changes in the line fluxes of [Fe III] and $[\mathrm{Fe} \mathrm{II}]$ is presented in Appendix C).

Many large-scale changes are readily identifiable as due to photo-ionization or expansion.

\section{B1 Changes due to photoionization}

(i) The [Fe III] flux fades across the low-ionization state (Fig. B1, $\phi=13.004$ to 13.032 ). Both lines are brightest in the -60 and $-20 \mathrm{~km} \mathrm{~s}^{-1}$ velocity slices, which bound the $-45 \mathrm{~km} \mathrm{~s}^{-1}$ peak velocity of the Weigelt objects (Zethson et al. 2012) and related debris associable with ejecta from the 1890s outburst (Weigelt \& Kraus 2012).

(ii) As [Fe III] disappears, these structures reappear in [Fe II] (Fig. B2, $\phi=13.004$ to 12.032).

(iii) Irregular [Fe III] structures to the northeast and north that come and go. Many shift with time in a clockwise direction (Fig. B1, -140 to $+20 \mathrm{~km} \mathrm{~s}^{-1}$ ). (iv) Long spikes of [Fe III] extending to the northeast and southwest appear and disappear at $-140 \mathrm{~km} \mathrm{~s}^{-1}$ as $\eta$ Car enters and leaves the low-ionization state. These spikes bound [Fe II] emission at several phases (Fig. B1, $\phi=12.163$, $12.977,13.073$ to 13.114 ).

(v) Numerous [Fe II] clumps appear at red-shifted velocities exceeding $+60 \mathrm{~km} \mathrm{~s}^{-1}$. Most of these clumps are 0.5 radially distant from $\eta$ Car.

(vi) The outermost structures disappear across the 5.7year interval of observations likely due to photo-ionization effects.

(vii) Both lines are brightest in the -60 and $-20 \mathrm{~km} \mathrm{~s}^{-1}$ velocity slices, which bound the $-45 \mathrm{~km} \mathrm{~s}^{-1}$ velocity of the Weigelt objects (Zethson et al. 2012) and related debris associable with the ejecta from the 1890s outburst. However the $[\mathrm{Fe}$ III] emission shrinks to the stellar position at all velocities across the 2014.6 periastron event at $\phi=13.004$ and 13.032. A very faint, diffuse halo persists extending 0. . 4 to the northwest. By contrast the [Fe II] strengthens at all velocities across the periastron event.

\section{B2 Changes due to expansion}

(i) The $[\mathrm{Fe}$ III] arcuate structures, displaced about 0. . 4 to the northwest and south of $\eta$ Car, appear by $\phi=12.163$ in the -60 to $-300 \mathrm{~km} \mathrm{~s}^{-1}$ velocity frames and expand radially through $\phi=12.977$ (Fig. B1). These same arcs appear in [Fe II] by $\phi=12.922$, brighten and persist across the lowionization state, then fade by $\phi=13.071$.

(ii) A single [Fe III] arc, displaced about 0 .' 2 to the northeast, appears by $\phi=12.163$ in the +140 to $+340 \mathrm{~km} \mathrm{~s}^{-1}$ iso-velocity frames and expands until fading by $\phi=12.977$ (Fig. B1). This arc also appears faintly in [Fe II] across the low-ionization state (Fig. B2).

(iii) Multiple pairs of arcs appear in [Fe II] to the west and south by $\phi=12.315$ and are traced through $\phi=13.071$. These pairs of arcs, described by Teodoro et al. (2013), expand radially from the $\eta$ Car position at initial separations in $\phi=12.315$ of 0. ' 2 and 0. .' 45 . When modeled as shells, the characteristic expansion velocity of these red-shifted shells is about $470 \mathrm{~km} \mathrm{~s}^{-1}$, somewhat larger than the terminal velocity, $v_{\infty, A}=420 \mathrm{~km} \mathrm{~s}^{-1}$ determined by Groh et al. (2012).

\section{APPENDIX C: THE CHANGING FLUXES OF [FE III] 4659 AND [FE II] 4815Å}

Damineli et al. (2008a) measured the binary period using Xray, optical and near infrared observations to be $2022.74 \pm 1.3$ days, referencing the zero phase of cycle 11 to JD 2452819.8 based upon the periodic disappearance of the narrow component to the He I $6678 \AA$ line. The 2014.6 drop in He I $6678 \AA$ was forecast to occur on JD 2456865.2. Teodoro et al. (2016) modeled the behavior of He II $4686 \AA$ across the three most recent spectroscopic minima, and determined that periastron occurred on JD $2456874.4 \pm 1.3$ days, or 9.2 days after the disappearance of the narrow component. Teodoro et al. (2016) found the best fit to the period to be $2022.7 \pm 0.3$ days. In this discussion to be consistent with published phase convention, we choose to use the zero phase, as defined by Damineli et al. (2008a) namely, $\phi=.0000$ is when the narrow component of the He I $6678 \AA$ disappears. However we 
keep in mind that periastron is now thought to occur 9.2 days, or $\delta \phi=0.0045$ later. Modeling of the X-ray hardness ratio also agrees with periastron being about nine days after disappearance of the He I narrow component (Corcoran et al. in prep).

Consistent with the behavior of He I and highly ionized species (Damineli et al. 2008a), we use the definition of the high-ionization state to be when both strong broad and narrow components of [Fe III] are present, which lasts for about 5.2 years of the 5.54-year period (see Fig. C1). The low-ionization state, defined by the interval when the narrow component of the high-ionization lines is absent, persists for several months. However, a weak, broad component of the high-ionization species, including $\mathrm{Fe}^{++}$, remains. The broad, weak component is isolated to the stellar core and a very diffuse region extending to the northwest as seen in the image slices across the low-ionization state in Fig. B1.

From our mappings, measures of these components are plotted in Fig. C1. The flux distributions with velocity at apastron are plotted for the [Fe III] $4659 \AA$ and the [Fe II]

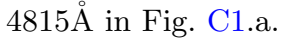

All other subplots in Fig. C1 display integrated fluxes plotted against orbital phase. Changes are greatest across the low-ionization state. In Fig. C1.b, [Fe II] (red) drops as [Fe III] (black) increases with the onset of the highionization state (12.084 to 12.163 and 13.071 to 13.114 ), and [Fe II] increases as [Fe III] fades as the low-ionization state approaches (12.922 to 13.004). Separation into a total broad components, -450 to $-100 \mathrm{~km} \mathrm{~s}^{-1}$ plus +100 to $+450 \mathrm{~km} \mathrm{~s}^{-1}$, leads to less pronounced changes (Figs. C1.c). The narrow, blue-shifted components, -100 to $0 \mathrm{~km} \mathrm{~s}^{-1}$ (Fig. C1.d) , show the largest changes across the lowionization state. The narrow, red-shifted components, 0 to $+100 \mathrm{~km} \mathrm{~s}^{-1}$, follow much weaker, changes (Fig. C1.e).

The blue-shifted, broad component, -450 to -100 $\mathrm{km} \mathrm{s}^{-1}$ (Fig. C1.f), shows a pronounced contrast between high-low-ionization state and low-ionization states, with the [Fe II] line being very faint during the high-ionization state. The change from high- to low-ionization state is much less pronounced for the red-shifted broad component, +100 to $+450 \mathrm{~km} \mathrm{~s}^{-1}$ (Fig. C1.g), partially due to the weak [Fe II] and Fe II emission-line contamination contributed to [Fe III] in the +300 to $+450 \mathrm{~km} \mathrm{~s}^{-1}$ velocity interval (see Fig. C3).

We demonstrate that the red-shifted component of [Fe III] is contaminated in two ways: i) structures seen at $+380 \mathrm{~km} \mathrm{~s}^{-1}$ for the [Fe II], [Fe III] and [N II] lines (see Section $\mathrm{C} 1)$ and ii) spectra of selected regions as seen in $[\mathrm{Fe}$ III] (Section C2).

\section{C1 Changes in structure by ionization potential, IP}

Velocity slices at -40 and $+380 \mathrm{~km} \mathrm{~s}^{-1}$ are presented in Fig. C2 for [Fe II] (.a), [Fe III] (.b) and [N II] (.c). The hook structure noted in Section 3.3.1 and Figs. 2 and 3 is always present in $-40 \mathrm{~km} \mathrm{~s}^{-1}$ images of [Fe II], but disappears across the low-ionization state in [Fe III]. Indeed across the low-ionization state, the broad component of [Fe III] is in the stellar core region and extends faintly to the northwest.

Comparison to the $[\mathrm{Fe} \mathrm{II}]$ and $[\mathrm{N} \mathrm{II}]$ velocity slices across the velocity range shows that [Fe II] strengthens across the low-ionization state at red-shifted velocities, but $[\mathrm{N} \mathrm{II}]$ vir-
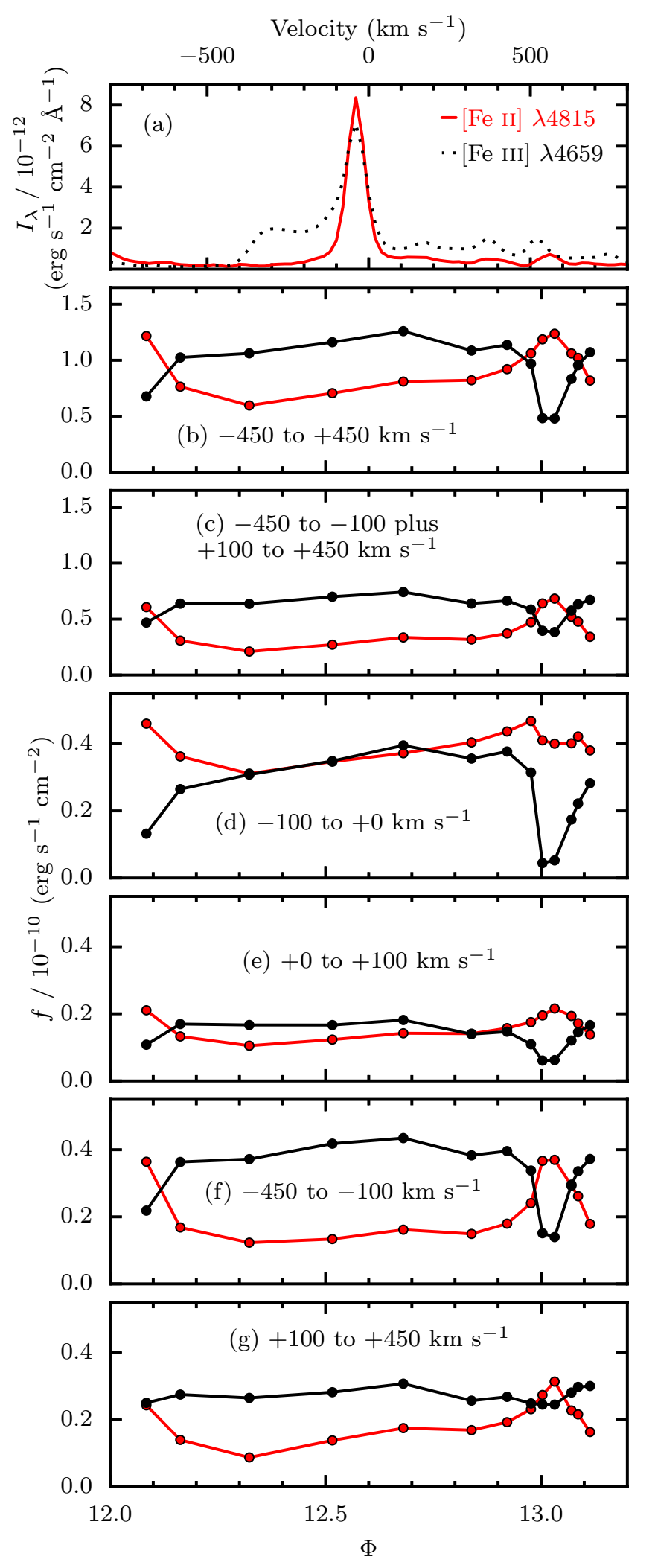

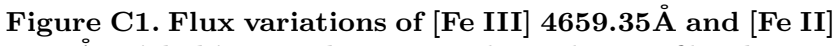
4815Å with binary phase. a: Velocity line profiles. b:- g: Integrated fluxes versus orbital phase. b: Total line flux from -450 to $+450 \mathrm{~km} \mathrm{~s}^{-1}$. c: Broad component excluding -100 to $+100 \mathrm{kms}, \mathbf{d}:-100$ to $0 \mathrm{~km} \mathrm{~s}^{-1}$ component, which includes the Weigelt objects, e: 0 to +100 component, f: -450 to $-100 \mathrm{~km} \mathrm{~s}^{-1}$ component, g: +100 to $+450 \mathrm{~km} \mathrm{~s}^{-1}$ component. 

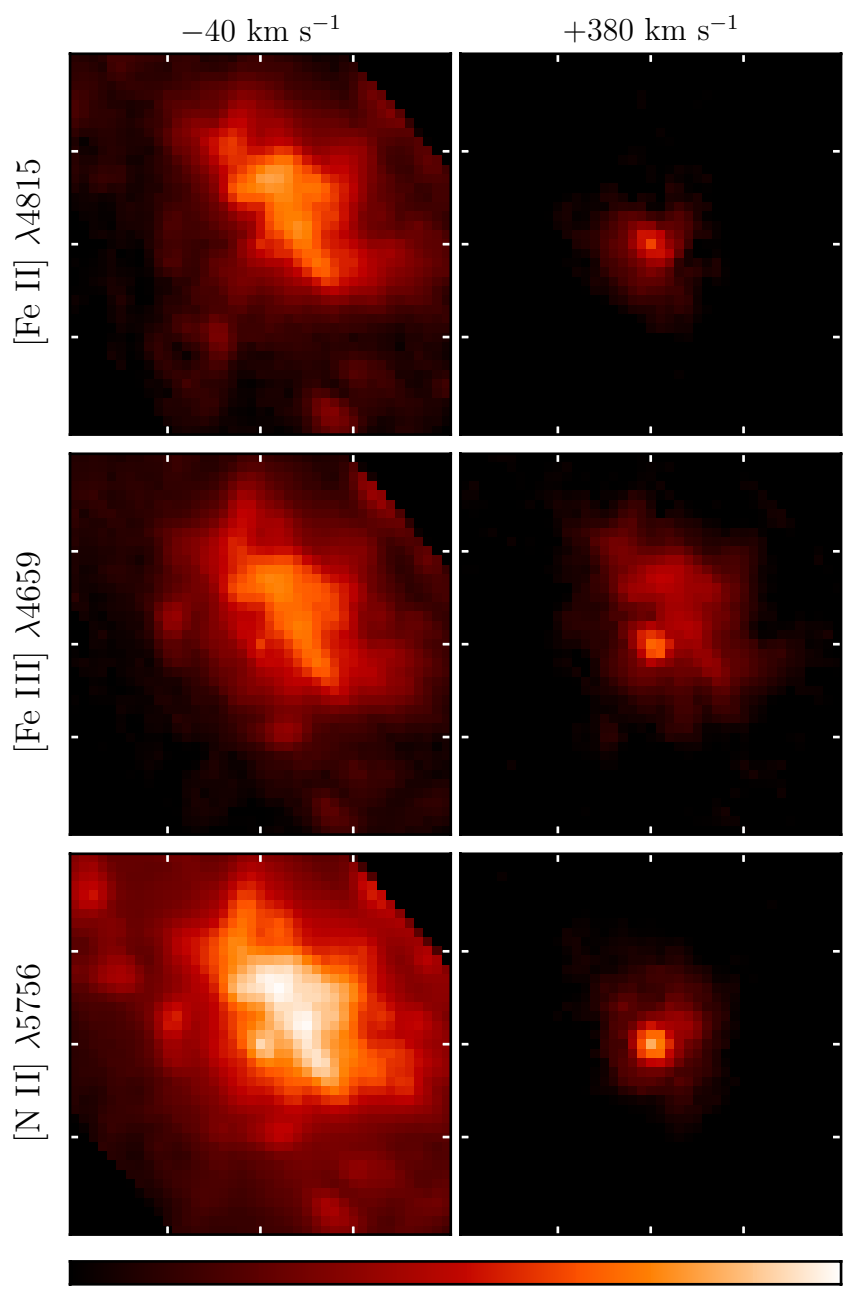

$$
\begin{array}{lcc}
-14 & -13 & -12 \\
& \log _{10}\left(I_{\lambda}\right)\left(\operatorname{erg~s}^{-1} \mathrm{~cm}^{-2} \AA^{-1}\right)
\end{array}
$$

Figure C2. Demonstration that the $+380 \mathrm{~km} \mathrm{~s}^{-1}$ [Fe III] images are contaminated by weak [Fe II] and Fe II line emission. Top row: [Fe II] $4815 \AA$. Middle row: [Fe III] $4659 \AA$. Bottom row: [N II] $5756 \AA$. Note that the hook-shaped structure appears in all three images centered at $-40 \mathrm{~km} \mathrm{~s}^{-1}$ (left column), and faintly in the [Fe III] $+380 \mathrm{~km} \mathrm{~s}^{-1}$ image. However the hook is not present in the [Fe II] and [N II] $+380 \mathrm{~km} \mathrm{~s}^{-1}$ images. Likewise the hook is not present in an $[\mathrm{Ar} \mathrm{III}]+380$ image (not shown) taken during the same mapping visit. Since the ionization potential, IP, of $\mathrm{N}^{+}$and $\mathrm{Ar}^{++}$are below and above the IP of $\mathrm{Fe}^{++}$, the structure seen in the [Fe III] $+380 \mathrm{~km} \mathrm{~s}^{-1}$ image must be contamination by two weak lines: Fe II $4665.01 \AA$ and [Fe II] 4665.75. . All iso-velocity, $40 \mathrm{~km} \mathrm{~s}^{-1}$-wide images are from mappings recorded in June, 2014 at phase, $\phi=12.922$. Mappings in [N II] required a separate grating setting, $5734 \AA$, and hence an additional CVZ orbit. As a result, [N II] mappings were done only at selected phases when deemed scientifically necessary.

tually disappears. At $+380 \mathrm{~km} \mathrm{~s}^{-1}$ only a faint core emission exists at the stellar core position. Yet a hook-shaped structure persists in the [Fe III] at this velocity, peaking at about $+380 \mathrm{~km} \mathrm{~s}^{-1}$. Likewise, no hook-shaped emission is present in the several [Ar III] $7137 \AA$ maps recorded at selected times during the orbital cycle. Since the hook-shaped structure does not exist for $\mathrm{N}^{+}$nor $\mathrm{Ar}^{++}$emissions with
$\mathrm{IP}=14.5,27.6 \mathrm{eV}$, then the hook-shaped structures must be attributed to the [Fe II] $4665.75 \AA$ and $\mathrm{Fe} 4665.01 \AA$ lines.

\section{C2 Velocity variations of [Fe III] for selected positions}

The forbidden emission varies considerably from point to point across the fossil winds. We demonstrate this for [Fe III] near apastron in Fig. C3 by presenting three spectral extractions from representative locations: $\mathrm{P} 1$, in the direction of a blue-shifted arcs expanding to the west-northwest, P2, to the north-northeast at the position of Weigelt D, a slowly moving emission clump thought to have originated during the 1890s event, and P3, a red-shifted clump almost due east of $\eta$ Car.

The fluxes for each spectrum are plotted on the same intensity scale. Likewise, the image slices are displayed on the same $\log$ scale. The narrow velocity component, -40 $\mathrm{km} \mathrm{s}^{-1}$, is brightest from the slowly moving gas, which includes Weigelt D. Yet weaker emission extends at red-shifted velocities that are not of [Fe III] origin. The peak near +375 $\mathrm{km} \mathrm{s}^{-1}$ originates from a blend of weak [Fe II] $4665.75 \AA$ and Fe II $4665.01 \AA$ and the peak at $510 \mathrm{~km} \mathrm{~s}^{-1}$ is due to a weaker [Fe III] 4668.06 (Zethson et al. 2012). The blend at $+375 \mathrm{~km} \mathrm{~s}^{-1}$ changes with orbital phase similar to the [Fe II] $4815.88 \AA$ and the $+510 \mathrm{~km} \mathrm{~s}^{-1}$ emission appears and disappears similar to the [Fe III] $4659.35 \AA$ emission. Note that in Fig. B1 a flux-adjusted, velocity-shifted blend of the [Fe II] $4815.88 \AA$ emission was subtracted to minimize its presence in the [Fe III] emission. The [Fe III] $4668.01 \AA$ emission was not subtracted, so it appears in the $+500 \mathrm{~km} \mathrm{~s}^{-1}$ velocity images.

We examined the spatial-velocity cubes of $[\mathrm{N}$ II $]$ and [Ar III] recorded at selected times during this program. Both have no identified narrow-line emission in the 0 to +600 $\mathrm{km} \mathrm{s}^{-1}$ velocity ranges (Zethson et al. 2012). Neither emission line shows the hook-shaped structure. Indeed the [N II] in the red-shifted velocity frames show a converging shell that disappears beyond $+420 \mathrm{~km} \mathrm{~s}^{-1}$. Hence the subtraction of the [Fe II] from the [Fe III] mosaic (Fig. B1) appears to be reasonably accurate and successful.

At $\mathrm{P} 1$, the $-40 \mathrm{~km} \mathrm{~s}^{-1}$ emission has dropped twentyfold, and a comparable flux is present extending to -200 $\mathrm{km} \mathrm{s}^{-1}$ (see Fig. 4). Red-shifted emission is considerably weaker.

At $\mathrm{P} 3$, while the $-40 \mathrm{~km} \mathrm{~s}^{-1}$ narrow emission is strongest, a narrow-line emission pops up at $+200 \mathrm{~km} \mathrm{~s}^{-1}$. The structural extent of this $+200 \mathrm{~km} \mathrm{~s}^{-1}$ emission is very different from the $-40 \mathrm{~km} \mathrm{~s}^{-1}$ emission (see Fig. 7).

This paper has been typeset from a $\mathrm{T}_{\mathrm{E}} \mathrm{X} / \mathrm{LAT}_{\mathrm{E}} \mathrm{X}$ file prepared by the author. 


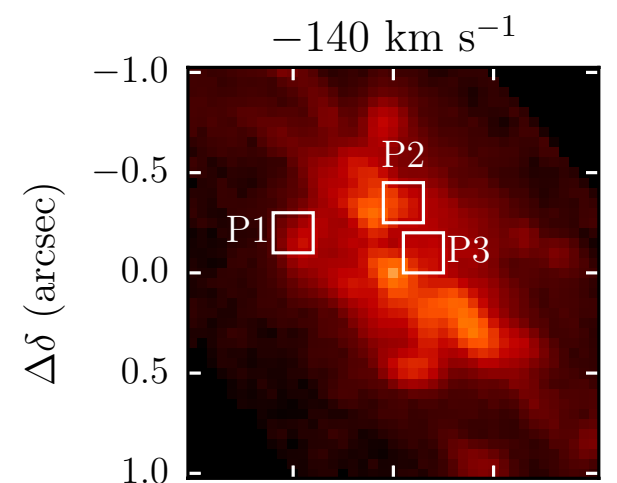

$\begin{array}{lll}0.5 & 0.0 & -0.5\end{array}$

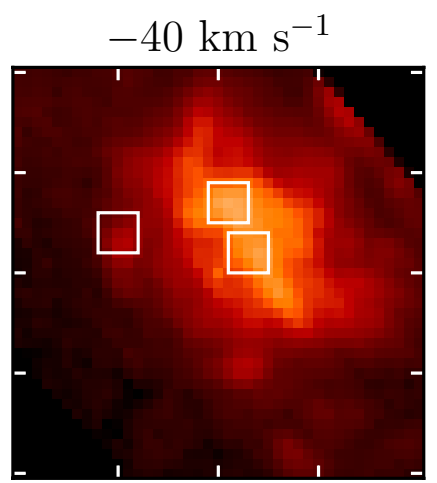

$\begin{array}{lll}0.5 & 0.0 & -0.5\end{array}$

$\Delta \alpha(\operatorname{arcsec})$

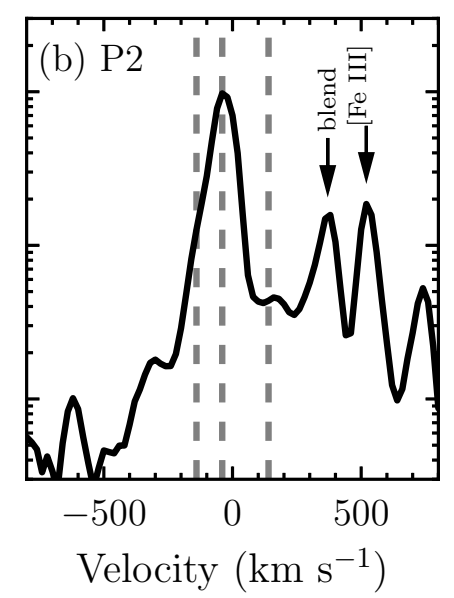

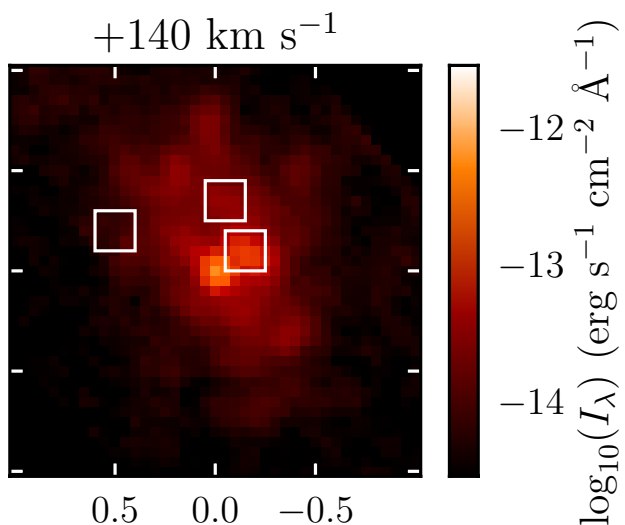

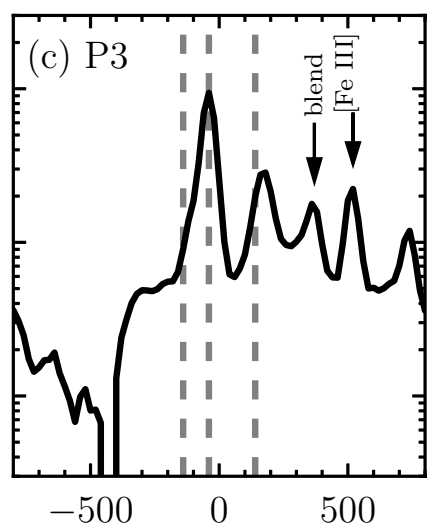

$-500 \quad 0 \quad 500$

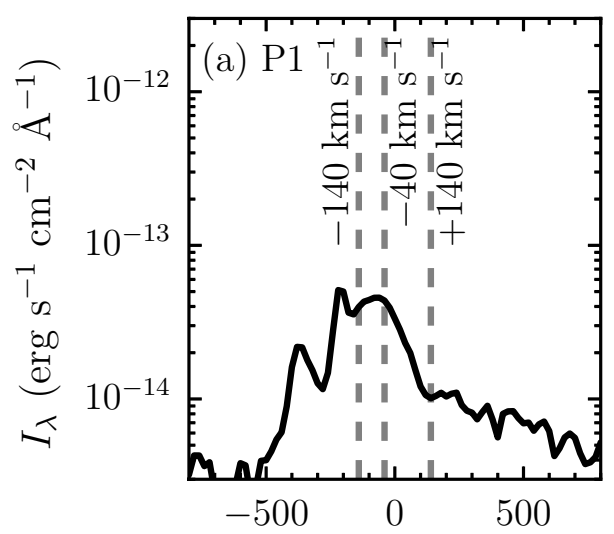

Figure C3. Sample spectra from selected portions of the interacting winds. Top row: Image slices $40 \mathrm{~km} \mathrm{~s}^{-1}$ wide centered at $-140,-40$ and $+140 \mathrm{~km} \mathrm{~s}^{-1}$. Bottom row: Extracted spectra from $0^{\prime \prime} 1 \times 00^{\prime \prime} 1$ areas. Left: Spectrum extracted near the peak of the $-140 \mathrm{~km} \mathrm{~s}^{-1}$ component, P1, to the west of $\eta$ Car. Center: Spectrum extracted at the peak of emission, P2, associable with Weigelt D. Right: Spectrum extracted at the peak of a red-shifted [Fe II] arc, P3. The data presented in this figure were recorded November 20, 2011 at $\eta$ Car's orbital phase, $\phi=12.516$. 